\title{
Green Function Formulism for Electromagnetic Wave Generated in Nanostructured Metamaterial of Finite Thickness: Isotropy and Anisotropy
}

\author{
Shunbo Li, ${ }^{1}$ Xiao Xiao, ${ }^{1}$ Bo Hou, ${ }^{2}$ and Weijia Wen ${ }^{1}$ \\ ${ }^{1}$ Department of Physics, The Hong Kong University of Science and Technology, Clear Water Bay, Kowloon, Hong Kong \\ ${ }^{2}$ School of Physical Science and Technology, Suzhou University, No. 1 Shizi Street, Jiangsu, Suzhou 215006, China \\ Correspondence should be addressed to Weijia Wen, phwen@ust.hk
}

Received 31 May 2012; Accepted 2 July 2012

Academic Editor: Zhaolin Lu

Copyright (C) 2012 Shunbo Li et al. This is an open access article distributed under the Creative Commons Attribution License, which permits unrestricted use, distribution, and reproduction in any medium, provided the original work is properly cited.

\begin{abstract}
A Green function formulism is developed to calculate the electromagnetic fields generated by sources embedded in nanostructured medium which could be represented by an effective electric permittivity tensor with finite thicknesses. The method begins with the decomposition of the generated mode into the eigenmodes in the medium, which have definite dispersions. To account the interface effect at boundaries, especially the mode conversion at the interface between anisotropic media, mode expansion method is combined into the theoretical framework. Thus, the electromagnetic wave in any given position can be gotten clearly. The formulism can provide conveniences of studying the novel properties of nanostructured metamaterials.
\end{abstract}

\section{Introduction}

Artificially constructed metamaterials $[1,2]$ have unique electromagnetic properties which cannot be obtained in natural materials, such as artificial magnetism, negative refraction [3], and near-field focusing [4]. Recently, active research areas related to metamaterials attracted much attention [5, 6], for instance, high/low epsilon metamaterials, designer dispersion, transformation optics metamaterials, switchable metamaterials, amplifying metamaterials, sensor metamaterials, nonlinear metamaterials, and quantum metamaterials [7]. The origin of novel property of structured metamaterial comes from the modification of effective permittivity and permeability. There are many developed methods to determine the effective permittivity and permeability $[8,9]$. Nanostructured metamaterials have wide applications in real world, such as manipulation of thermal radiation [10], plasmonic biosensor [11], artificial magnetic material [12], and superlens imaging [13]. Patterned metal at the interface between two media will generate surface-plasmon polaritons which alter surface waves and subsequently change radiative heat transfer, coherence properties, and Casimir forces [14].
To obtain the electromagnetic property of a nanostructured metamaterial, one would have to calculate Green function of the given system $[15,16]$. The conventional formulism [17] can handle homogeneous isotropic media very well, and the interface effects can be trivially accounted by introducing Fresnel coefficients. However, a lot of metamaterials exhibit anisotropy properties due to the special arrangement of structure [18]. Thus, the story becomes quite complex, when anisotropy of media is introduced. One distinguishing phenomenon of anisotropic media is the mode conversion $[19,20]$ happening at the interface between anisotropic media. Under the situation, the previous formulism is no longer suitable and convenient, since the mode conversion leads to an infinite multiplication for the Fresnel transmission and reflection coefficients. Thus, to study the coherence property of thermally radiative field from anisotropic media consisted by subwavelength structures with finite thickness, we have firstly to build up a general Green function theory working well in both isotropic media and anisotropic media. Exactly the motivation drives us to the present work. Since the development of metamaterial and nanooptics paves the way to design innovative structures, we herein proposed a Green function 


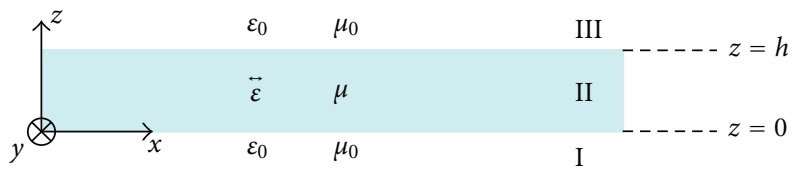

FIGURE 1: Schematic illustration of the geometry of the system under considering shows the thickness $(h)$ of the anisotropic (isotropic) layer and the coordinate system.

method to calculate electromagnetic wave generated by a structured metamaterials which have definite permittivity and permeability. In the following sections, we will firstly present our formulism for an arbitrary anisotropic medium with finite thickness in Section 2. To prove the correction of the method, we apply it to an isotropic medium case to recover the results in [17] in the same section. In Section 3, the Green functions linking spatial fields and sources in uniaxial and biaxial medium of finite thickness are calculated by following the general formulism shown in Section 2. A brief conclusion remark is made in the last section.

\section{Theoretical Formulism and Its Correction}

To begin with, we consider a piece of anisotropic medium with a finite thickness $h$ placed in vacuum (Figure 1), so that random sources locate only in the anisotropic medium. In the present work, it is assumed the relative permittivity tensor is anisotropic but its relative permeability is isotropic (the current formulism can be applied to the case that both permittivity and permeability are anisotropic, but we stay at the current situation to achieve a better demonstration of the formulism), and they can be written as:

$$
\overleftrightarrow{\mathcal{\varepsilon}}=\left(\begin{array}{ccc}
\varepsilon_{1} & 0 & 0 \\
0 & \varepsilon_{2} & 0 \\
0 & 0 & \varepsilon_{3}
\end{array}\right), \quad \mu=\mu .
$$

The relative permittivity and relative permeability of vacuum are equal to 1 . Once the information is given, for a given frequency the electromagnetic property is totally governed by Maxwell equations [17]:

$$
\begin{gathered}
\nabla \cdot\left(\overleftrightarrow{\varepsilon}_{r} \cdot \vec{E}(\vec{r})\right)=-4 \pi \nabla \cdot \vec{P}(\vec{r}), \\
\nabla \overrightarrow{\times} B(\vec{r})+i \widetilde{\omega} \mu_{r} \overleftrightarrow{\varepsilon}_{r} \cdot \vec{E}(\vec{r})=-i 4 \pi \tilde{\omega} \mu_{r} \vec{P}(\vec{r}) \\
+4 \pi \mu_{r} \nabla \times \vec{M}(\vec{r}) \\
\nabla \cdot \vec{B}(\vec{r})=0 \\
\nabla \times \vec{E}(\vec{r})-i \widetilde{\omega} \vec{B}(\vec{r})=0,
\end{gathered}
$$

where $\overleftrightarrow{\varepsilon}_{r}$ represents the relative permittivity tensor in different regions shown in Figure 1, $\mu_{r}$ means the permeability in different regions, $\tilde{\omega}$ represents $\omega / c$ (angular frequency over velocity of light in vacuum), $\vec{P}(\vec{r})$ denotes the random electric dipole source distribution in space, and $\vec{M}(\vec{r})$ is for random magnetic dipole source distribution.

2.1. Propagation Property in Homogeneous Medium. One can extract the information about the eigen-plane-wave modes and their corresponding dispersion relations by doing operations on the homogeneous Maxwell equations (dropping the source terms about $\vec{P}$ and $\vec{M}$ ), and one has

$$
\nabla \times(\nabla \times \vec{E}(\vec{r}))-\widetilde{\omega}^{2} \mu_{r} \overleftrightarrow{\varepsilon}_{r} \cdot \vec{E}(\vec{r})=0 .
$$

Taking into account the plane wave ansatz, we can have the matrix equation determining the eigenmodes for given regions:

$$
\begin{gathered}
\left(\begin{array}{ccc}
\tilde{\omega}^{2} \varepsilon_{x} \mu_{r}-k_{y}^{2}-k_{z}^{2} & k_{x} k_{y} & k_{x} k_{z} \\
k_{x} k_{y} & \tilde{\omega}^{2} \varepsilon_{y} \mu_{r}-k_{x}^{2}-k_{z}^{2} & k_{y} k_{z} \\
k_{x} k_{z} & k_{y} k_{z} & \tilde{\omega}^{2} \varepsilon_{z} \mu_{r}-k_{y}^{2}-k_{x}^{2}
\end{array}\right) \\
\times\left(\begin{array}{c}
E_{x} \\
E_{y} \\
E_{z}
\end{array}\right)=0,
\end{gathered}
$$

where $\varepsilon_{x}=\varepsilon_{1}, \varepsilon_{y}=\varepsilon_{2}, \varepsilon_{z}=\varepsilon_{3}$ in region II, $\varepsilon_{x}=\varepsilon_{y}=\varepsilon_{z}=$ 1 in regions I and III, $\mu_{r}=\mu$ in region II, and $\mu_{r}=1$ in regions I and III. For a nontrivial mode in the medium, the determinant of the coefficient matrix in (4) should vanish. Taking the homogeneous form of the first Maxwell equation in (2) as a constraint, the equation determining eigenmodes is arrived at

$$
\begin{aligned}
\widetilde{\omega}^{4}- & \widetilde{\omega}^{2}\left(\frac{k_{x}^{2}+k_{y}^{2}}{\varepsilon_{z} \mu_{r}}+\frac{k_{x}^{2}+k_{z}^{2}}{\varepsilon_{y} \mu_{r}}+\frac{k_{z}^{2}+k_{y}^{2}}{\varepsilon_{x} \mu_{r}}\right) \\
& +\left(\frac{k_{x}^{2}}{\varepsilon_{y} \varepsilon_{z} \mu_{r}^{2}}+\frac{k_{y}^{2}}{\varepsilon_{x} \varepsilon_{z} \mu_{r}^{2}}+\frac{k_{z}^{2}}{\varepsilon_{x} \varepsilon_{y} \mu_{r}^{2}}\right) \\
& \times\left(k_{x}^{2}+k_{z}^{2}+k_{y}^{2}\right)=0 .
\end{aligned}
$$

From (5) $k_{z}\left(\tilde{\omega}, k_{x}, k_{y}\right)$ can be solved out as the function of $\tilde{\omega}, k_{x}$ and $k_{y}$, and there are 4 solutions for $k_{z}$, namely, $\left\{k_{z, 1},-k_{z, 1} ; k_{z, 2},-k_{z, 2}\right\} \quad\left(k_{z, 1}\right.$ and $k_{z, 2}$ are positive real or positive imaginary numbers to denote the wave propagating or decaying along the positive $z$ direction.). To get the corresponding polarizations of the eigenmodes, we can solve (4) with the help of the homogeneous form of the first Maxwell equation in (2) and the corresponding dispersion relation of the given eigenmode $k_{z, i}^{2}-\left[k_{z, i}\left(\widetilde{\omega}, k_{x}, k_{y}\right)\right]^{2}=0$, where the index $i$ is 1 (2), when we solve the corresponding polarization of the first (second) eigenmode. For the convenience, we denote the unit vectors of them by $\hat{e}_{1, \pm}$ and $\hat{e}_{2, \pm}$, and conveniently the corresponding unit vectors of electric displacement vectors and magnetic field vectors are gotten by $\hat{d}_{i, \pm}=\overleftrightarrow{\varepsilon}_{r} \cdot \hat{e}_{i, \pm} /\left|\overleftrightarrow{\varepsilon}_{r} \cdot \hat{e}_{i, \pm}\right|$ and $\hat{h}_{i, \pm}=\vec{k}_{ \pm} \times$ $\hat{e}_{i, \pm} /\left|\vec{k}_{ \pm} \times \hat{e}_{i, \pm}\right|$. Then physics of propagating wave in the medium is quite clear: for propagating wave in the medium with arbitrary polarization its electric displacement vector is always perpendicular to wave vector; from the previous 
analysis, the electric displacement vector can be always decomposed in the plane perpendicular to the wave vector $\vec{k}$ into two components along $\hat{d}_{1}$ and $\hat{d}_{2}$, respectively. These two components obey different but definite dispersions, once the three parameters $\left(\tilde{\omega}, k_{x}\right.$, and $\left.k_{y}\right)$ are known.

2.2. Treatment of Sources. We now restore the source terms in Maxwell equations so that sources enter the picture. The analysis in the last section illuminates that the property of propagating modes in one medium is totally clear, when any three parameters of $\tilde{\omega}, k_{x}, k_{y}$, and $k_{z}$ are determined. On the other hand, when the spatial distribution and time evolution of the random sources are known, Fourier transformation can always help us to count the modes in a desired way (since we have limited in a given frequency case, $\widetilde{\omega}$ is known now):

$$
\begin{aligned}
\vec{P}(\vec{r}) & =\sum_{i=1,2} \int d z_{0} e^{\mp i k_{z, i} z_{0}} \int \frac{d \vec{k}}{(2 \pi)^{3}} \vec{P}_{\vec{k}_{i}} e^{i \overrightarrow{k_{i}} \cdot \vec{r}} \\
& =\int d z_{0} \int \frac{d \vec{\kappa}}{(2 \pi)^{2}} \delta\left(z-z_{0}\right) \vec{P}_{\vec{\kappa}} e^{i \vec{\kappa} \cdot \vec{R}}, \\
\vec{M}(\vec{r}) & =\sum_{i=1,2} \int d z_{0} e^{ \pm i k_{z,} z_{0}} \int \frac{d \vec{k}}{(2 \pi)^{3}} \vec{M}_{\vec{k}_{i}} e^{i \vec{k}_{i} \cdot \vec{r}} \\
& =\int d z_{0} \int \frac{d \vec{\kappa}}{(2 \pi)^{2}} \delta\left(z-z_{0}\right) \vec{M}_{\vec{\kappa}} e^{i \vec{\kappa} \cdot \vec{R}},
\end{aligned}
$$

where $z_{0} \in[0, h]$, since there should be no random sources in vacuum. Here and below it is convenient to put $\vec{R}=$ $(x, y)$. Such a transformation has clear physical meanings: the modes generated from sources are counted according to their tangential wave vectors, and to make the classification possible the price of cutting the medium into planes along $z$ direction is paid. The advantage of the classification is obvious: for each mode generated we can decompose it into two eigenmodes with definite dispersion relations and the same tangential wave vector.

Similarly, the electromagnetic field of a given position in space can be expressed as

$$
\begin{aligned}
& \vec{E}(\vec{R}, z)=\int \frac{d \vec{\kappa}}{(2 \pi)^{2}} \vec{E}(\vec{\kappa}, z) \exp (i \vec{\kappa} \cdot \vec{R}), \\
& \vec{B}(\vec{R}, z)=\int \frac{d \vec{\kappa}}{(2 \pi)^{2}} \vec{B}(\vec{\kappa}, z) \exp (i \vec{\kappa} \cdot \vec{R}),
\end{aligned}
$$

where

$$
\begin{aligned}
\vec{E}(\vec{\kappa}, z)= & \int d z_{0} \overleftrightarrow{G}_{E P}\left(\vec{\kappa} ; z-z_{0}\right) \vec{P}\left(\vec{\kappa}, z_{0}\right) \\
& +\int d z_{0} \overleftrightarrow{G}_{E M}\left(\vec{\kappa} ; z-z_{0}\right) \vec{M}\left(\vec{\kappa}, z_{0}\right), \\
\vec{B}(\vec{\kappa}, z)= & \int d z_{0} \overleftrightarrow{G}_{B P}\left(\vec{\kappa} ; z-z_{0}\right) \vec{P}\left(\vec{\kappa}, z_{0}\right) \\
& +\int d z_{0} \overleftrightarrow{G}_{B M}\left(\vec{\kappa} ; z-z_{0}\right) \vec{M}\left(\vec{\kappa}, z_{0}\right)
\end{aligned}
$$

In (8), $\overleftrightarrow{G}_{E P}, \overleftrightarrow{G}_{B P}, \overleftrightarrow{G}_{B M}$, and $\overleftrightarrow{G}_{B M}$ are Green functions linking the field in space with the source for a given tangential wave vector $\vec{\kappa}$. Once getting the relation between fields in space and source, the forms of Green functions are determined spontaneously. Because of the linearity of Maxwell equation, we can focus to one mode with tangential wave vector $\vec{\kappa}$ generated from a source plane labeled as $z_{0}$, and to account all modes one needs only to integrate all $\vec{\kappa}$ and $z$ dimensions of the anisotropic medium.

2.3. Mode Expansion in Each Region. To determine the field generated from source plate locating at $z_{0}$ with tangential wave vector $\vec{\kappa}$, the mode expansion method would be found useful $[21,22]$. Since we have assumed the anisotropy of permeability and isotropy of permittivity, mode expansion according to magnetic field may be more convenient, and they are demonstrated in Figure 2. For the given frequency $\tilde{\omega}$ and tangential wave vector $\vec{\kappa}$, the fields in region II can be expanded as

$$
\begin{aligned}
& \vec{B}_{\mathrm{II}}(\widetilde{\omega}, \vec{\kappa})=\left[\vec{B}_{1, g,+} \exp \left(-i k_{z, 1} z_{0}\right)+\vec{B}_{2, g,+} \exp \left(-i k_{z, 2} z_{0}\right)\right] \\
& \times \Theta\left(z-z_{0}\right)+\vec{B}_{1, b,+}+\vec{B}_{2, b,+} \\
& +\left[\vec{B}_{1, g,-} \exp \left(i k_{z, 1} z_{0}\right)+\vec{B}_{2, g,-} \exp \left(i k_{z, 2} z_{0}\right)\right] \\
& \times \Theta\left(z_{0}-z\right)+\vec{B}_{1, b,-}+\vec{B}_{2, b,-} \\
& +\vec{B} \delta\left(z-z_{0}\right) \exp (i \vec{\kappa} \cdot \vec{R}) \text {, } \\
& \vec{D}_{\mathrm{II}}(\tilde{\omega}, \vec{\kappa})=\left[\vec{D}_{1, g,+} \exp \left(-i k_{z, 1} z_{0}\right)+\vec{D}_{2, g,+} \exp \left(-i k_{z, 2} z_{0}\right)\right] \\
& \times \Theta\left(z-z_{0}\right)+\vec{D}_{1, b,+}+\vec{D}_{2, b,+} \\
& +\left[\vec{D}_{1, g,-} \exp \left(i k_{z, 1} z_{0}\right)+\vec{D}_{2, g,-} \exp \left(i k_{z, 2} z_{0}\right)\right] \\
& \times \Theta\left(z_{0}-z\right)+\vec{D}_{1, b,-}+\vec{D}_{2, b,-} \\
& +\vec{D} \delta\left(z-z_{0}\right) \exp (i \vec{\kappa} \cdot \vec{R}) \text {, } \\
& \vec{E}_{\mathrm{II}}(\widetilde{\omega}, \vec{\kappa})=\left[\vec{E}_{1, g,+} \exp \left(-i k_{z, 1} z_{0}\right)+\vec{E}_{2, g,+} \exp \left(-i k_{z, 2} z_{0}\right)\right] \\
& \times \Theta\left(z-z_{0}\right)+\vec{E}_{1, b,+}+\vec{E}_{2, b,+} \\
& +\left[\vec{E}_{1, g,-} \exp \left(i k_{z, 1} z_{0}\right)+\vec{E}_{2, g,-} \exp \left(i k_{z, 2} z_{0}\right)\right] \\
& \times \Theta\left(z_{0}-z\right)+\vec{E}_{1, b,-}+\vec{E}_{2, b,-} \\
& +\vec{E} \delta\left(z-z_{0}\right) \exp (i \vec{\kappa} \cdot \vec{R})
\end{aligned}
$$




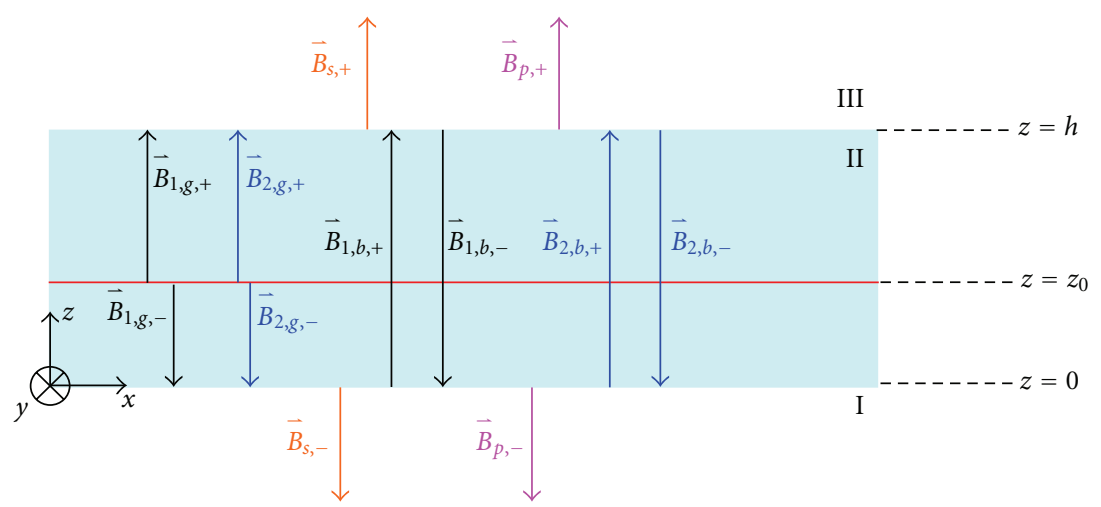

FIGURE 2: Schematic illustration of eigenmode expansion in three regions: $\vec{B}_{s,-}$ and $\vec{B}_{p,-}$ denote the eigen-plane-wave modes in region I, and the minus sign is to denote the direction of propagating is along $z$-direction; $\vec{B}_{1, g, \pm}$ in region II denotes the first eigenmode generated from the source plane, $\vec{B}_{2, g, \pm}$ is to denote the second eigenmode generated from the source plane, $\vec{B}_{1, b, \pm}$ in region II expresses the first eigenmode bouncing back at the upper and lower boundaries, and $\vec{B}_{2, b, \pm}$ gives the second eigenmode bouncing back at the upper and lower boundaries (the plus and minus signs denotes the propagating along $z+$ and $z$-directions); $\vec{B}_{s,+}$ and $\vec{B}_{p,+}$ denote the eigen-plane wave modes in region III, and the minus sign is to denote the direction of propagating is along $z+$ direction.

where

$$
\begin{gathered}
\vec{B}_{i, g, \pm}=B_{i, g, \pm} \exp (i \vec{\kappa} \cdot \vec{R}) \exp \left( \pm i k_{z, i} z\right) \hat{h}_{i, \pm}, \\
\vec{B}_{i, b, \pm}=B_{i, b, \pm} \exp (i \vec{\kappa} \cdot \vec{R}) \exp \left( \pm i k_{z, i} z\right) \hat{h}_{i, \pm}, \\
\vec{D}_{i, g, \pm}=\frac{-1}{i \tilde{\omega} \mu} \nabla \times \vec{B}_{i, g, \pm}, \\
\vec{E}_{i, g, \pm}=\overleftrightarrow{\varepsilon}-1 \cdot \vec{D}_{i, g, \pm} \\
\vec{D}_{i, b, \pm}=\frac{-1}{i \widetilde{\omega} \mu} \nabla \times \vec{B}_{i, b, \pm}, \\
\vec{E}_{i, b, \pm}=\overleftrightarrow{\varepsilon}-1 \cdot \vec{D}_{i, b, \pm} .
\end{gathered}
$$$$
i=1,2 \text {, }
$$

$\vec{B}(\vec{D}, \vec{E})$ is to account the local (nonpropagating) field locating on the source plane, and $\Theta\left(z-z_{0}\right)$ is step function to denote the directions of modes launching from the source plane. In regions I and III, the eigenmodes are s- and ppolarized modes, and field can be expanded as

$$
\begin{aligned}
\vec{B}_{\mathrm{I}}(\tilde{\omega}, \vec{\kappa})= & \left(B_{s,-} \hat{h}_{s,-}^{\mathrm{I}}+B_{p,-} \hat{h}_{p,-}^{\mathrm{I}}\right) \\
& \times \exp (i \vec{\kappa} \cdot \vec{R}) \exp \left(-i k_{z, \mathrm{I}} z\right), \\
\vec{D}_{\mathrm{I}}(\widetilde{\omega}, \vec{\kappa})= & \vec{E}_{\mathrm{I}}(\widetilde{\omega}, \vec{\kappa}) \\
= & \left(B_{s,-} \hat{e}_{s,-}^{\mathrm{I}}+B_{p,-} \hat{e}_{p,-}^{\mathrm{I}}\right) \\
& \times \exp (i \vec{\kappa} \cdot \vec{R}) \exp \left(-i k_{z, \mathrm{I}} z\right), \\
\vec{B}_{\mathrm{III}}(\tilde{\omega}, \vec{\kappa})= & \left(B_{s,+} \hat{h}_{s,+}^{\mathrm{III}}+B_{p,+} \hat{h}_{p,+}^{\mathrm{III}}\right) \\
& \times \exp (i \vec{\kappa} \cdot \vec{R}) \exp \left(i k_{z, \mathrm{III}} z\right),
\end{aligned}
$$

$$
\begin{aligned}
\vec{D}_{\mathrm{III}}(\tilde{\omega}, \vec{\kappa})= & \vec{E}_{\mathrm{III}}(\tilde{\omega}, \vec{\kappa}) \\
= & \left(B_{s,+} \hat{e}_{s,+}^{\mathrm{II}}+B_{p,+} \hat{e}_{p,+}^{\mathrm{III}}\right) \\
& \times \exp (i \vec{\kappa} \cdot \vec{R}) \exp \left(i k_{z, \mathrm{III}} z\right),
\end{aligned}
$$

where the unit vectors are defined as

$$
\begin{gathered}
\hat{e}_{s, \pm}^{\alpha}=\frac{\vec{k}_{s, \pm} \times \hat{z}}{\left|\vec{k}_{s, \pm} \times \hat{z}\right|}=\left(\begin{array}{c}
\kappa \cos \theta \\
\kappa \sin \theta \\
\pm k_{z}
\end{array}\right) \times\left(\begin{array}{l}
0 \\
0 \\
1
\end{array}\right)=\left(\begin{array}{c}
\sin \theta \\
-\cos \theta \\
0
\end{array}\right), \\
\hat{h}_{s, \pm}^{\alpha}=\frac{\vec{k}_{s, \pm} \times \hat{e}_{s, \pm}}{\left|\vec{k}_{s, \pm} \times \hat{e}_{s, \pm}\right|}=\frac{1}{\widetilde{\omega} \sqrt{\varepsilon_{r} \mu_{r}}}\left(\begin{array}{c} 
\pm k_{z} \cos \theta \\
\pm k_{z} \sin \theta \\
-\kappa
\end{array}\right), \\
\hat{h}_{p, \pm}^{\alpha}=\frac{\vec{k}_{p, \pm} \times \hat{z}}{\left|\vec{k}_{p, \pm} \times \hat{z}\right|}=\left(\begin{array}{c}
\kappa \cos \theta \\
\kappa \sin \theta \\
\pm k_{z}
\end{array}\right) \times\left(\begin{array}{c}
0 \\
0 \\
1
\end{array}\right)=\left(\begin{array}{c}
\sin \theta \\
-\cos \theta \\
0
\end{array}\right), \\
\hat{e}_{p, \pm}^{\alpha}=\frac{-\vec{k}_{p, \pm} \times \hat{h}_{p, \pm}}{\left|\vec{k}_{p, \pm} \times \hat{h}_{p, \pm}\right|}=\frac{1}{\widetilde{\omega} \sqrt{\varepsilon_{r} \mu_{r}}}\left(\begin{array}{c}
\mp k_{z} \cos \theta \\
\mp k_{z} \sin \theta \\
\kappa
\end{array}\right),
\end{gathered}
$$

where $\alpha=$ I, II, III denotes the region where the unit vectors belong.

2.4. Determination of Generated Field in Each Region and Green Functions. The amplitudes of the four eigenmodes 
launching from source plane for the given frequency $\widetilde{\omega}$ and tangential wave vector $\vec{\kappa}$ can be determined by putting the fields in region II into the second and the last Maxwell equations in (2). The propagating modes in the mode expansion fulfill homogeneous Maxwell equation, and thus the electric fields and the magnetic fields of these propagating modes cancel. The second and the last Maxwell equations in (2), respectively, read

$$
\begin{aligned}
& \delta\left(z-z_{0}\right) \hat{z} \\
& \times\left[\vec{B}_{1, g,+} \exp \left(-i k_{z, 1} z_{0}\right)+\vec{B}_{2, g,+} \exp \left(-i k_{z, 2} z_{0}\right)\right. \\
& \left.-\vec{B}_{1, g,-} \exp \left(i k_{z, 1} z_{0}\right)-\vec{B}_{2, g,-} \exp \left(i k_{z, 2} z_{0}\right)\right] \\
& +\hat{z} \times \vec{B} \delta^{\prime}\left(z-z_{0}\right) \exp (i \vec{\kappa} \cdot \vec{R})+i \vec{\kappa} \\
& \times \vec{B} \exp (i \vec{\kappa} \cdot \vec{R}) \delta\left(z-z_{0}\right) \\
& +i \tilde{\omega} \mu \overleftrightarrow{\varepsilon} \cdot \vec{E} \delta\left(z-z_{0}\right) \exp (i \vec{\kappa} \cdot \vec{R}) \\
& =-4 \pi i \tilde{\omega} \mu \vec{P} \delta\left(z-z_{0}\right) \exp (i \vec{\kappa} \cdot \vec{R})+4 \pi \mu i \vec{\kappa} \\
& \times \vec{M} \exp (i \vec{\kappa} \cdot \vec{R}) \delta\left(z-z_{0}\right) \\
& +4 \pi \mu \delta^{\prime}\left(z-z_{0}\right) \hat{z} \times \vec{M} \exp (i \vec{\kappa} \cdot \vec{R}), \\
& \delta\left(z-z_{0}\right) \hat{z} \\
& \times\left[\vec{E}_{1, g,+} \exp \left(-i k_{z, 1} z_{0}\right)-\vec{E}_{1, g,-} \exp \left(i k_{z, 1} z_{0}\right)\right. \\
& \left.+\vec{E}_{2, g,+} \exp \left(-i k_{z, 2} z_{0}\right)-\vec{E}_{2, g,-} \exp \left(i k_{z, 2} z_{0}\right)\right] \\
& +\hat{z} \times \vec{E} \delta^{\prime}\left(z-z_{0}\right) \exp (i \vec{\kappa} \cdot \vec{R})+i \vec{\kappa} \\
& \times \vec{E} \exp (i \vec{\kappa} \cdot \vec{R}) \delta\left(z-z_{0}\right) \\
& =i \widetilde{\omega} \vec{B} \delta\left(z-z_{0}\right) \exp (i \vec{\kappa} \cdot \vec{R}),
\end{aligned}
$$

where we have used $\nabla \Theta\left(z-z_{0}\right)=\hat{z} \delta\left(z-z_{0}\right)$ and $\nabla \delta\left(z-z_{0}\right)=$ $\hat{z} \delta^{\prime}\left(z-z_{0}\right)$. By requiring the coefficients in front of $\delta\left(z-z_{0}\right)$ and $\delta^{\prime}\left(z-z_{0}\right)$ equal for the two sides, the amplitudes $B_{i, g, \pm}$ can be solved out. The fields generated in the regions outside of the medium can be determined by imposing the condition of continuity of tangential field at two boundaries $(z=0$ and $z=h)$ :

$$
\begin{aligned}
\vec{E}_{\text {II }}(\tilde{\omega}, \vec{\kappa} ; z=0) \cdot(\hat{x} \hat{x}+\hat{y} \hat{y}) \\
\quad=\left(B_{s,-} \hat{e}_{s,-}+B_{p,-} \hat{e}_{p,-}\right) \cdot(\hat{x} \hat{x}+\hat{y} \hat{y}) \exp (i \vec{\kappa} \cdot \vec{R}),
\end{aligned}
$$

$$
\begin{aligned}
& \vec{B}_{\mathrm{II}}(\widetilde{\omega}, \vec{\kappa} ; z=0) \cdot(\hat{x} \hat{x}+\hat{y} \hat{y}) \\
& \quad=\left(B_{s,-} \hat{h}_{s,-}+B_{p,-} \hat{h}_{p,-}\right) \cdot(\hat{x} \hat{x}+\hat{y} \hat{y}) \exp (i \vec{\kappa} \cdot \vec{R}),
\end{aligned}
$$

for $z=0$ boundary, where $\hat{x}$ and $\hat{y}$ are the unit vectors of $x$ and $y$ directions,

$$
\begin{aligned}
& \vec{E}_{\mathrm{II}}(\widetilde{\omega}, \vec{\kappa} ; z=h) \cdot(\hat{x} \hat{x}+\hat{y} \hat{y}) \\
& =\left(B_{s,+} \hat{e}_{s,+}+B_{p,+} \hat{e}_{p,+}\right) \cdot(\hat{x} \hat{x}+\hat{y} \hat{y}) \exp (i \vec{\kappa} \cdot \vec{R}) \exp (i k h), \\
& \vec{B}_{\mathrm{II}}(\widetilde{\omega}, \vec{\kappa} ; z=h) \cdot(\hat{x} \hat{x}+\hat{y} \hat{y}) \\
& =\left(B_{s,+} \hat{h}_{s,+}+B_{p,+} \hat{h}_{p,+}\right) \cdot(\hat{x} \hat{x}+\hat{y} \hat{y}) \exp (i \vec{\kappa} \cdot \vec{R}) \exp (i k h),
\end{aligned}
$$

for $z=h$ boundary.

2.5. Correction of the Formulism: Isotropic Case. To prove the correction of the formulism, in this subsection we apply it to isotropic medium and compare the results gotten with well-known results in [17]. Equations from (13) to (16) are solved under the current case to get the Green functions. Since now the relative permittivity in region II then reduces to a scalar (we denote it by $\varepsilon$ ), the eigenmodes in the region are also $s$ - and $p$-polarized modes. The dispersions for $s$ - and $p$-polarized modes are the same and given as $\widetilde{\omega}^{2} \varepsilon \mu=\kappa^{2}+k_{z}^{2}$ in region II and $\widetilde{\omega}^{2}=\kappa^{2}+k_{z}^{2}$ in region I and III. The unit vectors of electric field and magnetic field are given in (12). The further convenience is that $\hat{e}_{s^{ \pm}}, \hat{\kappa}$ and $\hat{z}$ build up a righthand coordinate, which can simplify the solution of (13) and (14) by decomposing the fields according to the three directions. After equating the coefficients in front of $\delta^{\prime}\left(z-z_{0}\right)$ in (13) and (14), the following relations are found:

$$
\begin{gathered}
\hat{z} \times \vec{B}=4 \pi \mu \hat{z} \times \vec{M}, \\
\hat{z} \times \vec{E}=0 .
\end{gathered}
$$

The local magnetic source relates with local magnetic field as $B_{\hat{e}_{s \pm}}=4 \pi \mu M_{\hat{e}_{s \pm}}$ and $B_{\widehat{\kappa}}=4 \pi \mu M_{\hat{\kappa}}$ and $\hat{e}_{s, \pm}$ and $\hat{\kappa}$ components of local electric field vanish $\left(E_{\hat{e}_{s \pm}}=E_{\widehat{\kappa}}=0\right)$. From the equality of the coefficients in front of $\delta\left(z-z_{0}\right)$, we can get the other equations, and we write them according to the components along $\hat{e}_{s \pm}, \hat{\kappa}$, and $\hat{z}$ directions for (13), and (14) respectively,

$$
\begin{gathered}
\frac{1}{\sqrt{\varepsilon \mu}}\left(B_{s, g,+}+B_{s, g,-}\right)=\frac{4 \pi i \widetilde{\omega}^{2} \mu}{k_{z}} P_{\hat{e}_{s, \pm}}-\frac{4 \pi \mu i \kappa \tilde{\omega}}{k_{z}} M_{\hat{z}}, \\
B_{p, g,+}-B_{p, g,-}=-4 \pi i \tilde{\omega} \mu P_{\widehat{\kappa}}, \\
i \omega \varepsilon \mu E_{\widehat{z}}=-i 4 \pi \tilde{\omega} \mu P_{\widehat{z}}, \\
\frac{1}{\sqrt{\mu \varepsilon}}\left(B_{p, g,+}+B_{p, g,-}\right)+\frac{\kappa k_{\mathrm{II}}}{k_{z, \mathrm{II}}}=\frac{i \widetilde{\omega} k_{\mathrm{II}}}{k_{z, \mathrm{II}}} B_{\hat{e}_{s, \pm}}, \\
\frac{1}{\sqrt{\mu \varepsilon}}\left(B_{s, g,+}-B_{s, g,-}\right)=i \widetilde{\omega} B_{\widehat{\kappa}}, \\
B_{\widehat{z}}=0 .
\end{gathered}
$$


The fields launching from the source can be gotten by solving (18) and the results are collected as follows:

$$
\begin{aligned}
& B_{s, g,+}=\left(\frac{2 \pi i \tilde{\omega}^{2} \mu}{k_{z, \mathrm{II}}} \sqrt{\mu \varepsilon}\left(\hat{e}_{s, \pm}^{\mathrm{I}} \cdot \vec{P}\right)+\frac{2 \pi \mu i \tilde{\omega}^{2} \mu \varepsilon}{k_{z, \mathrm{II}}}\left(\hat{h}_{s,+}^{\mathrm{II}} \cdot \vec{M}\right)\right), \\
& B_{s, g,-}=\left(\frac{2 \pi i \tilde{\omega}^{2} \mu}{k_{z, \mathrm{II}}} \sqrt{\mu \varepsilon}\left(\hat{e}_{s, \pm}^{\mathrm{II}} \cdot \vec{P}\right)+\frac{2 \pi \mu i \tilde{\omega}^{2} \mu \varepsilon}{k_{z, \mathrm{II}}}\left(\hat{h}_{s,-}^{\mathrm{II}} \cdot \vec{M}\right)\right), \\
& B_{p, g,+}=\left(\frac{2 \pi i \mu \tilde{\omega} k_{\mathrm{II}}}{k_{z, \mathrm{II}}} \sqrt{\varepsilon \mu}\left(\hat{e}_{s, \pm}^{\mathrm{II}} \cdot \vec{M}\right)-\frac{2 \pi i \tilde{\omega} k_{\mathrm{II}} \mu}{k_{z, \mathrm{II}}}\left(\hat{h}_{s,+}^{\mathrm{II}} \cdot \vec{P}\right)\right), \\
& B_{p, g,-}=\left(\frac{2 \pi i \mu \tilde{\omega} k_{\mathrm{II}}}{k_{z, \mathrm{II}}} \sqrt{\varepsilon \mu}\left(\hat{e}_{s, \pm}^{\mathrm{II}} \cdot \vec{M}\right)-\frac{2 \pi i \tilde{\omega} k_{\mathrm{II}} \mu}{k_{z, \mathrm{II}}}\left(\hat{h}_{s,--}^{\mathrm{II}} \cdot \vec{P}\right)\right),
\end{aligned}
$$

where the results are written in a compact form with the help of the unit vectors defined and the same with (2.37) in [17], and thus Green functions in infinite bulk isotropic medium gotten by present method are correct.

To determine the fields in regions I and III, the boundary equations (15) and (16) should be solved. Isotropy of the medium in region II provides great simplification: since the orthogonality between tangential wave vectors $\hat{e}_{s, \pm}^{T}=\hat{h}_{p, \pm}^{T}$ and $\hat{h}_{s, \pm}^{T}=-\hat{e}_{p, \pm}^{T}$ always holds, it is not necessary to arrange the equations in $x-y-z$ coordinate. Taking the advantage, the boundary equation (15) for $z=0$ becomes

$$
\begin{aligned}
& B_{s,-} \hat{e}_{s,-}^{T, \mathrm{I}} \\
& =\frac{1}{\sqrt{\mu \varepsilon}}\left(B_{s, b,+} \hat{e}_{s,+}^{T, \mathrm{II}}+B_{s, g,-} \exp \left(i k_{z, \mathrm{II}} z_{0}\right) \hat{e}_{s,-}^{T, \mathrm{II}}+B_{s, b,-} \hat{e}_{s,-}^{T, \mathrm{II}}\right) \\
& B_{p,-} \hat{e}_{p,-}^{T, \mathrm{I}} \\
& \quad=\frac{1}{\sqrt{\mu \varepsilon}}\left(B_{p, b,+} \hat{e}_{p,+}^{T, \mathrm{II}}+B_{p, g,-} \exp \left(i k_{z, \mathrm{II}} z_{0}\right) \hat{e}_{p,-}^{T, \mathrm{II}}+B_{p, b,-} \hat{e}_{p,-}^{T, \mathrm{II}}\right), \\
& B_{p,-} \hat{h}_{p,-}^{T, \mathrm{I}} \\
& =B_{p, b,+} \hat{h}_{p,+}^{T, \mathrm{II}}+B_{p, g,-} \exp \left(i k_{z, \mathrm{II}} z_{0}\right) \hat{h}_{p,-}^{T, \mathrm{II}}+B_{p, b,-} \hat{h}_{p,-}^{T, \mathrm{II}}, \\
& B_{s,-} \hat{h}_{s,-}^{T, \mathrm{I}} \\
& \quad=B_{s, b,+} \hat{h}_{s,+}^{T, \mathrm{II}}+B_{s, g,-} \exp \left(i k_{z, \mathrm{II}} z_{0}\right) \hat{h}_{s,-}^{T, \mathrm{II}}+B_{s, b,-} \hat{h}_{s,-}^{T, \mathrm{II}} .
\end{aligned}
$$

Similarly, the boundary equation (16) for $z=h$ gives

$$
\begin{aligned}
B_{s,+} & \exp \left(i k_{z, \mathrm{I}} h\right) \hat{e}_{s,+}^{T, \mathrm{III}} \\
=\frac{1}{\sqrt{\mu \varepsilon}} & \left(B_{s, g,+} \exp \left[i k_{z, \mathrm{II}}\left(h-z_{0}\right)\right] \hat{e}_{s,+}^{T, \mathrm{II}}\right. \\
& +B_{s, b,+} \exp \left(i k_{z, \mathrm{II}} h\right) \hat{e}_{s,+}^{T, \mathrm{II}} \\
& \left.+B_{s, b,-} \exp \left(-i k_{z, \mathrm{II}} h\right) \hat{e}_{s,-}^{T, \mathrm{II}}\right)
\end{aligned}
$$

$$
\begin{aligned}
B_{p,+} & \exp \left(i k_{z, \mathrm{I}} h\right) \hat{e}_{p,+}^{T, \mathrm{III}} \\
= & \frac{1}{\sqrt{\mu \varepsilon}}\left(B_{p, g,+} \exp \left[i k_{z, \mathrm{II}}\left(h-z_{0}\right)\right] \hat{e}_{p,+}^{T, \mathrm{II}}\right. \\
& +B_{p, b,+} \exp \left(i k_{z, \mathrm{II}} h\right) \hat{e}_{p,+}^{T, \mathrm{II}} \\
& \left.+B_{p, b,-} \exp \left(-i k_{z, \mathrm{II}} h\right) \hat{e}_{p,-}^{T, \mathrm{II}}\right), \\
B_{p,+} & \exp \left(i k_{z, \mathrm{I}} h\right) \hat{h}_{p,+}^{T, \mathrm{III}} \\
= & B_{p, g,+} \exp \left[i k_{z, \mathrm{II}}\left(h-z_{0}\right)\right] \hat{h}_{p,+}^{T, \mathrm{II}} \\
& +B_{p, b,+} \exp \left(i k_{z, \mathrm{II}} h\right) \hat{h}_{p,+}^{T, \mathrm{II}}+B_{p, b,-} \exp \left(-i k_{z, \mathrm{II}} h\right) \hat{h}_{p,-}^{T, \mathrm{II}}, \\
B_{s,+} \exp \left(i k_{z, \mathrm{I}} h\right) \hat{h}_{s,+}^{T, \mathrm{III}} & \\
= & B_{s, g,+} \exp \left[i k_{z, \mathrm{II}}\left(h-z_{0}\right)\right] \hat{h}_{s,+}^{T, \mathrm{II}} \\
& +B_{s, b,+} \exp \left(i k_{z, \mathrm{II}} h\right) \hat{h}_{s,+}^{T, \mathrm{II}}+B_{s, b,-} \exp \left(-i k_{z, \mathrm{II}} h\right) \hat{h}_{s,-}^{T, \mathrm{II}},
\end{aligned}
$$

where $\hat{e}_{s, \pm}^{T, \mathrm{I}(\mathrm{III})}, \hat{e}_{s, \pm}^{T, \mathrm{II}}, \hat{e}_{p, \pm}^{T, \mathrm{I}(\mathrm{III})}, \hat{e}_{p, \pm}^{T, \mathrm{II}}, \hat{h}_{p, \pm}^{T, \mathrm{I}(\mathrm{III})}, \hat{h}_{p, \pm}^{T, \mathrm{II}}, \hat{h}_{s, \pm}^{T, \mathrm{I}(\mathrm{III})}, \hat{h}_{s, \pm}^{T, \mathrm{II}}$ are the tangential parts of these unit vectors in different regions and $k_{z, \text { II }}=k_{z, s}=k_{z, p}$. Once $B_{s,-}$ and $B_{p,-}\left(B_{s,+}\right.$ and $\left.B_{p,+}\right)$ are solved from (20) and (21), the generated fields in region I (III) from the source plane are determined. The fields in region I are given as follows:

$$
\begin{aligned}
& \vec{B}_{\mathrm{I}}(\widetilde{\omega}, \vec{\kappa}) \\
& =\frac{1}{\sqrt{\mu \varepsilon}}\left[\frac{r_{\mathrm{II}, \mathrm{I}}^{s} e^{i k_{z, \mathrm{II}} z_{0}}}{1-t_{\mathrm{II}, \mathrm{I}}^{s} e^{i 2 k_{z, \mathrm{II}} h}} B_{s, g,-}\right. \\
& \left.+\frac{r_{\mathrm{II}, \mathrm{I}}^{s} t_{\mathrm{II}, \mathrm{I}}^{s} e^{i k_{z, \mathrm{II}}\left(2 h-z_{0}\right)}}{1-t_{\mathrm{II}, \mathrm{I}}^{s} e^{i 2 k_{z, \mathrm{II}} h}} B_{s, g,+}\right] \\
& \times \hat{h}_{s,-} \exp (i \vec{\kappa} \cdot \vec{R}) \exp \left(-i k_{z, \mathrm{I}} z\right) \\
& +\frac{1}{\sqrt{\mu \varepsilon}}\left[\frac{r_{\mathrm{II}, \mathrm{I}}^{p} e^{i k_{z, \mathrm{II}} z_{0}}}{1-t_{\mathrm{II}, \mathrm{I}}^{p} e^{i 2 k_{z, \mathrm{II}} h}} B_{p, g,-}\right. \\
& \left.+\frac{r_{\mathrm{II}, \mathrm{I}}^{p} t_{\mathrm{II}, \mathrm{I}}^{p} e^{i k_{z, \mathrm{II}}\left(2 h-z_{0}\right)}}{1-t_{\mathrm{II}, \mathrm{I}}^{p} e^{i 2 k_{z, \mathrm{II}} h}} B_{p, g,+}\right] \\
& \times \hat{h}_{p,-} \exp (i \vec{\kappa} \cdot \vec{R}) \exp \left(-i k_{z, \mathrm{I}} z\right), \\
& \vec{E}_{\mathrm{I}}(\widetilde{\omega}, \vec{\kappa}) \\
& =\frac{1}{\sqrt{\mu \varepsilon}}\left[\frac{r_{\mathrm{II}, \mathrm{I}}^{s} e^{i k_{z, \mathrm{II}} z_{0}}}{1-t_{\mathrm{II}, \mathrm{I}}^{s} e^{i 2 k_{z, \mathrm{II}} h}} B_{s, g,-}\right. \\
& \left.+\frac{r_{\mathrm{II}, \mathrm{I}}^{s} t_{\mathrm{II}, \mathrm{I}}^{s} e^{i k_{z, \mathrm{II}}\left(2 h-z_{0}\right)}}{1-t_{\mathrm{II}, \mathrm{I}}^{s} e^{i 2 k_{z, \mathrm{II}} h}} B_{s, g,+}\right] \\
& \times \hat{e}_{s,-} \exp (i \vec{\kappa} \cdot \vec{R}) \exp \left(-i k_{z, \mathrm{I}} z\right) \\
& +\frac{1}{\sqrt{\mu \varepsilon}}\left[\frac{r_{\mathrm{II}, \mathrm{I}}^{p} e^{i k_{z, \mathrm{II}} z_{0}}}{1-t_{\mathrm{II}, \mathrm{I}}^{p} e^{i 2 k_{z, \mathrm{II}} h}} B_{p, g,-}\right. \\
& \left.+\frac{r_{\mathrm{II}, \mathrm{I}}^{p} t_{\mathrm{II}, \mathrm{e}}^{p} e^{i k_{z, \mathrm{II}}\left(2 h-z_{0}\right)}}{1-t_{\mathrm{II}, \mathrm{I}}^{p} e^{i 2 k_{z, \mathrm{II}} h}} B_{p, g,+}\right] \\
& \times \hat{e}_{p,-} \exp (i \vec{\kappa} \cdot \vec{R}) \exp \left(-i k_{z, \mathrm{I}} z\right) .
\end{aligned}
$$


International Journal of Optics

7

The fields generated in region III are gotten as

$$
\begin{aligned}
& \vec{B}_{\text {III }}(\widetilde{\omega}, \vec{\kappa}) \\
& =\frac{1}{\sqrt{\varepsilon \mu}}\left[\frac{r_{\mathrm{II}, \mathrm{III}}^{s} t_{\mathrm{II}, \mathrm{III}}^{s} e^{i k_{z, \mathrm{II}}\left(z_{0}+h\right)}}{1-t_{\mathrm{II}, \mathrm{III}}^{s} e^{2} e^{i 2 k_{z, \mathrm{II}} h}} B_{s, g,-}\right. \\
& \left.+\frac{r_{\mathrm{II}, \mathrm{III}}^{s} e^{i k_{z, \mathrm{II}}\left(h-z_{0}\right)}}{1-t_{\mathrm{II}, \mathrm{III}}^{s} e^{i 2 k_{z, \mathrm{II}} h}} B_{s, g,+}\right] \\
& \times \hat{h}_{s,+} e^{-i k_{z, \mathrm{I}} h} \exp (i \vec{\kappa} \cdot \vec{R}) \exp \left(i k_{z, \mathrm{III}} z\right) \\
& +\frac{1}{\sqrt{\varepsilon \mu}}\left[\frac{r_{\mathrm{II}, \mathrm{III}}^{p} t_{\mathrm{II}, \mathrm{III}}^{p} e^{i k_{z, \mathrm{II}}\left(z_{0}+h\right)}}{1-t_{\mathrm{II}, \mathrm{III}}^{p} e^{i 2 k_{z, \mathrm{II}} h}} B_{p, g,-}\right. \\
& \left.+\frac{r_{\mathrm{II}, \mathrm{III}}^{p} e^{i k_{z, \mathrm{II}}\left(h-z_{0}\right)}}{1-t_{\mathrm{II}, \mathrm{III}}^{p} e^{i 2 k_{z, \mathrm{II}} h}} B_{p, g,+}\right] \\
& \times \hat{h}_{p,+} e^{-i k_{z, \mathrm{I}} h} \exp (i \vec{\kappa} \cdot \vec{R}) \exp \left(i k_{z, \mathrm{III}} z\right), \\
& \vec{E}_{\text {III }}(\widetilde{\omega}, \vec{\kappa}) \\
& =\frac{1}{\sqrt{\varepsilon \mu}}\left[\frac{r_{\mathrm{II}, \mathrm{III}}^{s} t_{\mathrm{II}, \mathrm{III}}^{s} e^{i k_{z, \mathrm{II}}\left(z_{0}+h\right)}}{1-t_{\mathrm{II}, \mathrm{III}}^{s} e^{i 2 k_{z, \mathrm{II}} h}} B_{s, g,-}\right. \\
& \left.+\frac{r_{\mathrm{II}, \mathrm{III}}^{s} e^{i k_{z, \mathrm{II}}\left(h-z_{0}\right)}}{1-t_{\mathrm{II}, \mathrm{III}}^{s} e^{i 2 k_{z, \mathrm{II}} h}} B_{s, g,+}\right] \\
& \times \hat{e}_{s,+} e^{-i k_{z, \mathrm{I}} h} \exp (i \vec{\kappa} \cdot \vec{R}) \exp \left(i k_{z, \mathrm{III}} z\right) \\
& +\frac{1}{\sqrt{\varepsilon \mu}}\left[\frac{r_{\mathrm{II}, \mathrm{III}}^{p} t_{\mathrm{II}, \mathrm{III}}^{p} e^{i k_{z, \mathrm{II}}\left(z_{0}+h\right)}}{1-t_{\mathrm{II}, \mathrm{III}}^{p} e^{i 2 k_{z, \mathrm{II}} h}} B_{p, g,-}\right. \\
& \left.+\frac{r_{\mathrm{II}, \mathrm{III}}^{p} e^{i k_{z, \mathrm{II}}\left(h-z_{0}\right)}}{1-{t_{\mathrm{II}, \mathrm{III}}^{p}}^{2} e^{i 2 k_{z, \mathrm{II}} h}} B_{p, g,+}\right] \\
& \times \hat{e}_{p,+} e^{-i k_{z, \mathrm{I}} h} \exp (i \vec{\kappa} \cdot \vec{R}) \exp \left(i k_{z, \mathrm{III}} z\right),
\end{aligned}
$$

where $t_{i, j}^{s}=\left(k_{z, i}-k_{z, j}\right) /\left(k_{z, i}+k_{z, j}\right), t_{i, j}^{s}=2 k_{z, i} /\left(k_{z, i}+\right.$ $\left.k_{z, j}\right), t_{i, j}^{p}=\left(k_{z, i} \varepsilon_{j} \mu_{j}-k_{z, j} \varepsilon_{i} \mu_{i}\right) /\left(k_{z, i} \varepsilon_{j} \mu_{j}+k_{z, j} \varepsilon_{i} \mu_{i}\right), r_{i, j}^{p}=$ $2 k_{z, i} \sqrt{\varepsilon_{j} \mu_{j}} \sqrt{\varepsilon_{i} \mu_{i}} /\left(k_{z, i} \varepsilon_{j} \mu_{j}+k_{z, j} \varepsilon_{i} \mu_{i}\right)$ are Fresnel coefficients. Since $B_{s, g, \pm}$ and $B_{p, g, \pm}$ have been related with the sources, the fields in regions I and III in fact can spontaneously relate with the sources. Thus, Green functions linking the fields in these regions and source plane are ready to be gotten by following definition (8):

$$
\begin{aligned}
& \overleftrightarrow{G}_{B M}^{\mathrm{I}}\left(\vec{\kappa} ; z-z_{0}\right) \\
& =\frac{2 \pi \mu i \tilde{\omega} k_{\mathrm{II}}}{k_{z, \mathrm{II}}}\left(\frac{t_{\mathrm{II}, \mathrm{I}}^{s}\left(r_{\mathrm{II}, \mathrm{I}}^{s} e^{i k_{z, \mathrm{II}}\left(2 h-z_{0}\right)} \hat{h}_{s,-}^{\mathrm{I}} \hat{h}_{s,+}^{\mathrm{II}}+e^{i k_{z, \mathrm{II}} z_{0}} \hat{h}_{s,-}^{\mathrm{I}} \hat{h}_{s,-}^{\mathrm{II}}\right)}{1-r_{\mathrm{II}, \mathrm{I}}^{s} e^{i 2 k_{z, \mathrm{II}} h}}\right. \\
& \left.+\frac{t_{\mathrm{II}, \mathrm{I}}^{\mathrm{p}}\left(e^{i k_{z, \mathrm{II}} z_{0}}+r_{\mathrm{II}, \mathrm{I}}^{p} e^{i k_{z, \mathrm{II}}\left(2 h-z_{0}\right)}\right)}{1-r_{\mathrm{II}, \mathrm{I}}^{p} e^{i 2 k_{z, \mathrm{II}} h}} \hat{h}_{p,-}^{\mathrm{I}} \hat{e}_{s, \pm}^{\mathrm{II}}\right) \\
& \quad \times e^{i \vec{\kappa} \cdot \vec{R}} e^{-i k_{z, \mathrm{I}} z},
\end{aligned}
$$

$$
\begin{aligned}
& \overleftrightarrow{G}_{B P}^{\mathrm{I}}\left(\vec{\kappa} ; z-z_{0}\right) \\
& =\frac{2 \pi i \tilde{\omega}^{2} \mu}{k_{z, \mathrm{II}}} \\
& \times\left(\frac{t_{\mathrm{IIII}}^{s}\left(e^{i k_{z, \mathrm{II}} z_{0}}+r_{\mathrm{II}, \mathrm{I}}^{s} e^{i k_{z, \mathrm{II}}\left(2 h-z_{0}\right)}\right)}{1-r_{\mathrm{II}, \mathrm{I}}^{s} e^{i 2 k_{z, \mathrm{II}} h}} \hat{h}_{s,-}^{\mathrm{I}} \hat{e}_{s, \pm}^{\mathrm{II}}\right. \\
& \left.-\frac{t_{\mathrm{II}, \mathrm{I}}^{\mathrm{P}}\left(e^{i k_{z, \mathrm{II}} z_{0}} \hat{h}_{p,-}^{\mathrm{I}} \hat{h}_{s,-}^{\mathrm{II}}+r_{\mathrm{II}, \mathrm{I}}^{p} e^{i k_{z, \mathrm{II}}\left(2 h-z_{0}\right)} \hat{h}_{p,-}^{\mathrm{I}} \hat{h}_{s,+}^{\mathrm{II}}\right)}{1-r_{\mathrm{II}, \mathrm{I}}^{p} e^{i 2 k_{z, \mathrm{II}} h}}\right) \\
& \times e^{i \vec{\kappa} \cdot \vec{R}} e^{-i k_{z, 1} z}, \\
& \overleftrightarrow{G}_{E M}^{\mathrm{I}}\left(\vec{\kappa} ; z-z_{0}\right) \\
& =\frac{2 \pi \mu i \tilde{\omega} k_{\mathrm{II}}}{k_{z, \mathrm{II}}} \\
& \times\left[\frac{r_{\mathrm{II}, \mathrm{I}}^{s}\left(t_{\mathrm{II}, \mathrm{I}}^{s} e^{i k_{z, \mathrm{II}}\left(2 h-z_{0}\right)} \hat{e}_{s,-}^{\mathrm{I}} \hat{h}_{s,+}^{\mathrm{II}}+e^{i k_{z, \mathrm{II}} z_{0}} \hat{e}_{s,-}^{\mathrm{I}} \hat{h}_{s,-}^{\mathrm{II}}\right)}{1-t_{\mathrm{II}, \mathrm{I}}^{s} e^{i 2 k_{z, \mathrm{II}} h}}\right. \\
& \left.+\frac{r_{\mathrm{II}, \mathrm{I}}^{p}\left(e^{i k_{z, \mathrm{II}} z_{0}}+t_{\mathrm{II}, \mathrm{I}}^{p} e^{i k_{z, \mathrm{II}}\left(2 h-z_{0}\right)}\right)}{1-t_{\mathrm{II}, \mathrm{I}}^{p} e^{i 2 k_{z, \mathrm{II}} h}} \hat{e}_{p,-}^{\mathrm{I}} \hat{e}_{s, \pm}^{\mathrm{II}}\right] \\
& \times e^{i \vec{\kappa} \cdot \vec{R}} e^{-i k_{z, \mathrm{I}} z}, \\
& \overleftrightarrow{G}_{E M}^{\mathrm{I}}\left(\vec{\kappa} ; z-z_{0}\right) \\
& =\frac{2 \pi i \widetilde{\omega}^{2} \mu}{k_{z, \mathrm{II}}}\left[\frac{t_{\mathrm{II}, \mathrm{I}}^{s}\left(e^{i k_{z, \mathrm{II}} z_{0}}+r_{\mathrm{II}, \mathrm{I}}^{s} e^{i k_{z, \mathrm{II}}\left(2 h-z_{0}\right)}\right)}{1-r_{\mathrm{II}, \mathrm{I}}^{s} e^{i 2 k_{z, \mathrm{II}} h}} \hat{e}_{s,-}^{\mathrm{I}} \hat{e}_{s, \pm}^{\mathrm{I}}\right. \\
& \left.-\frac{t_{\mathrm{II}, \mathrm{I}}^{\mathrm{p}}\left(e^{i k_{z, \mathrm{II}} z_{0}} \hat{e}_{p,-}^{\mathrm{I}} \hat{h}_{s,-}^{\mathrm{II}}+r_{\mathrm{II}, \mathrm{I}}^{p} e^{i k_{z, \mathrm{II}}\left(2 h-z_{0}\right)} \hat{e}_{p,-}^{\mathrm{I}} \hat{h}_{s,+}^{\mathrm{II}}\right)}{1-r_{\mathrm{II}, \mathrm{I}}^{p^{2}} e^{i 2 k_{z, \mathrm{II}} h}}\right] \\
& \times e^{i \vec{\kappa} \cdot \vec{R}} e^{-i k_{z, 1} z}, \\
& \overleftrightarrow{G}_{B M}^{\text {III }}\left(\vec{\kappa} ; z-z_{0}\right) \\
& =\frac{2 \pi \mu i \tilde{\omega} k_{\mathrm{II}}}{k_{z, \mathrm{II}}} \\
& \times\left[\frac{t_{\mathrm{II}, \mathrm{III}}^{s}\left(r_{\mathrm{II}, \mathrm{III}}^{s} e^{i k_{z, \mathrm{II}}\left(z_{0}+h\right)} \hat{h}_{s,+}^{\mathrm{III}} \hat{h}_{s,-}^{\mathrm{II}}+e^{i k_{z, \mathrm{II}}\left(h-z_{0}\right)} \hat{h}_{s,+}^{\mathrm{III}} \hat{h}_{s,+}^{\mathrm{II}}\right)}{1-r_{\mathrm{II}, \mathrm{III}}{ }^{2} e^{i 2 k_{z, \mathrm{II}} h}}\right. \\
& \left.+\frac{t_{\mathrm{II}, \mathrm{III}}^{\mathrm{p}}\left(r_{\mathrm{II}, \mathrm{III}}^{p} e^{i k_{z, \mathrm{II}}\left(z_{0}+h\right)}+e^{i k_{z, \mathrm{II}}\left(h-z_{0}\right)}\right)}{1-r_{\mathrm{II}, \mathrm{III}}^{p} e^{2} e^{i 2 k_{z, \mathrm{II}} h}} \hat{h}_{p,+}^{\mathrm{III}} \hat{e}_{s, \pm}^{\mathrm{II}}\right] \\
& \times e^{i \kappa_{z, \text { III }}(z-h)} e^{i \vec{k} \cdot \vec{R}},
\end{aligned}
$$




$$
\begin{aligned}
& \overleftrightarrow{G}_{B P}^{I I I}\left(\vec{\kappa} ; z-z_{0}\right) \\
& =\frac{2 \pi i \tilde{\omega}^{2} \mu}{k_{z, \mathrm{II}}} \\
& \times\left[\frac{t_{\mathrm{II}, \mathrm{III}}^{s}\left(r_{\mathrm{II}, \mathrm{III}}^{s} e^{i k_{z, \mathrm{II}}\left(z_{0}+h\right)}+e^{i k_{z, \mathrm{II}}\left(h-z_{0}\right)}\right)}{1-r_{\mathrm{II}, \mathrm{III}}^{s} e^{i 2 k_{z, \mathrm{II}} h}} \hat{h}_{s,+}^{\mathrm{III}} \hat{e}_{s, \pm}^{\mathrm{II}}\right. \\
& \left.-\frac{t_{\mathrm{II}, \mathrm{III}}^{\mathrm{P}}\left(r_{\mathrm{II}, \mathrm{III}}^{\mathrm{p}} e^{i k_{z, \mathrm{II}}\left(z_{0}+h\right)} \hat{h}_{p,+}^{\mathrm{III}} \hat{h}_{s,-}^{\mathrm{II}}+e^{i k_{z, \mathrm{II}}\left(h-z_{0}\right)} \hat{h}_{p,+}^{\mathrm{III}} \hat{h}_{s,+}^{\mathrm{II}}\right)}{1-r_{\mathrm{II}, \mathrm{III}}^{p} e^{i 2 k_{z, \mathrm{II}} h}}\right] \\
& \times e^{i \kappa_{z, \mathrm{III}}(z-h)} e^{i \vec{k} \cdot \vec{R}} \\
& \overleftrightarrow{G}_{E M}^{\mathrm{III}}\left(\vec{\kappa} z-z_{0}\right) \\
& =\frac{2 \pi \mu i \tilde{\omega} k_{\mathrm{II}}}{k_{z, \mathrm{II}}} \\
& \times\left[\frac{t_{\mathrm{II}, \mathrm{III}}^{s}\left(r_{\mathrm{II}, \mathrm{III}}^{s} e^{i k_{z, \mathrm{II}}\left(z_{0}+h\right)} \hat{e}_{s,+}^{\mathrm{III}} \hat{h}_{s,-}^{\mathrm{II}}+e^{i k_{z, \mathrm{II}}\left(h-z_{0}\right)} \hat{e}_{s,+}^{\mathrm{III}} \hat{h}_{s,+}^{\mathrm{II}}\right)}{1-{r_{\mathrm{II}, \mathrm{III}}^{s}}^{2} e^{i 2 k_{z, \mathrm{II}} h}}\right. \\
& \left.+\frac{t_{\mathrm{II}, \mathrm{III}}^{\mathrm{p}}\left(r_{\mathrm{II}, \mathrm{III}}^{p} e^{i k_{z, \mathrm{II}}\left(z_{0}+h\right)}+e^{i k_{z, \mathrm{II}}\left(h-z_{0}\right)}\right)}{1-r_{\mathrm{II}, \mathrm{III}}^{p} e^{2} e^{i 2 k_{z, \mathrm{II}} h}} \hat{e}_{p,+}^{\mathrm{III}} \hat{e}_{s, \pm}^{\mathrm{II}}\right] \\
& \times e^{i \kappa_{z, \mathrm{III}}(z-h)} e^{i \vec{k} \cdot \vec{R}} \\
& \overleftrightarrow{G}_{E P}^{\mathrm{III}}\left(\vec{\kappa} z-z_{0}\right) \\
& =\frac{2 \pi i \widetilde{\omega}^{2} \mu}{k_{z, \mathrm{II}}} \\
& \times\left[\frac{t_{\mathrm{II}, \mathrm{III}}^{s}\left(r_{\mathrm{II}, \mathrm{III}}^{s} e^{i k_{z, \mathrm{II}}\left(z_{0}+h\right)}+e^{i k_{z, \mathrm{II}}\left(h-z_{0}\right)}\right)}{1-r_{\mathrm{II}, \mathrm{III}}^{s} e^{i 2 k_{z, \mathrm{II}} h}} \hat{e}_{s,+}^{\mathrm{III}} \hat{e}_{s, \pm}^{\mathrm{II}}\right. \\
& \left.-\frac{t_{\mathrm{II}, \mathrm{III}}^{p}\left(r_{\mathrm{II}, \mathrm{III}}^{p} e^{i k_{z, \mathrm{II}}\left(z_{0}+h\right)} \hat{e}_{p,+}^{\mathrm{III}} \hat{h}_{s,-}^{\mathrm{II}}+e^{i k_{z, \mathrm{II}}\left(h-z_{0}\right)} \hat{e}_{p,+}^{\mathrm{III}} \hat{h}_{s,+}^{\mathrm{II}}\right)}{1-r_{\mathrm{II}, \mathrm{III}}^{p} e^{i 2 k_{z, \mathrm{II}} h}}\right] \\
& \times e^{i \kappa_{z, \mathrm{III}}(z-h)} e^{i \vec{k} \cdot \vec{R}} .
\end{aligned}
$$

These results exactly recover (4.18) in [17], and thus the correction of current formulism is proved.

\section{Green Functions for Sources in Anisotropic Medium}

The formulism developed in the last section is then applied to obtain Green functions linking sources in anisotropic medium and fields in space. The advantage of mode expansion method would be obviously shown in the following study.
3.1. Sources in Uniaxial Crystal. Under the situation, the permittivity tensor in region II would be read as $\operatorname{diag}\left(\varepsilon_{o}, \varepsilon_{e}, \varepsilon_{o}\right)$, where without losing generality we have assumed the anisotropy is along $y$ direction (so that mode conversion still happens at the interface). Then the equation determining eigenmodes (5) becomes

$$
\left(\widetilde{\omega}^{2}-\frac{k_{x}^{2}}{\varepsilon_{o} \mu}-\frac{k_{y}^{2}}{\varepsilon_{o} \mu}-\frac{k_{z}^{2}}{\varepsilon_{o} \mu}\right)\left(\widetilde{\omega}^{2}-\frac{k_{x}^{2}}{\varepsilon_{e} \mu}-\frac{k_{y}^{2}}{\varepsilon_{o} \mu}-\frac{k_{z}^{2}}{\varepsilon_{e} \mu}\right)=0 .
$$

Thus, there are two dispersions gotten: the mode with dispersion $\tilde{\omega}^{2}-k_{x}^{2} / \varepsilon_{o} \mu-k_{y}^{2} / \varepsilon_{o} \mu-k_{z}^{2} / \varepsilon_{o} \mu=0$ is known as $o$-light, and the mode with dispersion $\tilde{\omega}^{2}-k_{x}^{2} / \varepsilon_{e} \mu-$ $k_{y}^{2} / \varepsilon_{o} \mu-k_{z}^{2} / \varepsilon_{e} \mu=0$ is known as $e$-light. The corresponding unit vector for electric field can be solved from (4) and the constraint (the homogeneous form of the first Maxwell equation in (2)) and given as

$$
\begin{aligned}
\hat{e}_{o, \pm}=\frac{1}{\sqrt{\kappa^{2} \cos ^{2} \theta+k_{z, o}^{2}}}\left(\begin{array}{c} 
\pm k_{z, o} \\
0 \\
-\kappa \cos \theta
\end{array}\right) & \sqrt{\frac{\left(\kappa^{2} \cos ^{2} \theta+k_{z, e}^{2}\right)}{\varepsilon_{e}^{2}}+\frac{\kappa^{2} \sin ^{2} \theta}{\varepsilon_{o}^{2}}} \sqrt{\kappa^{2} \cos ^{2} \theta+k_{z, e}^{2}} \\
& \times\left(\begin{array}{c}
\varepsilon_{o}^{-1} \kappa^{2} \sin \theta \cos \theta \\
\pm\left(-k_{z, e}^{2}-\kappa^{2} \cos { }^{2} \theta\right) \\
\pm \varepsilon_{o}^{-1} k_{z, e} \kappa \sin \theta
\end{array}\right)
\end{aligned}
$$

Thus, we can have the unit vectors for magnetic field and electric displacement vector as

$$
\begin{gathered}
\hat{h}_{o \pm}=\frac{1}{k_{o} \sqrt{\kappa^{2} \cos ^{2} \theta+k_{z, o}^{2}}}\left(\begin{array}{c}
-\kappa^{2} \sin \theta \cos \theta \\
k_{z, o}^{2}+\kappa^{2} \cos ^{2} \theta \\
\mp k_{z, o} \kappa \sin \theta
\end{array}\right), \\
\hat{h}_{e \pm}=\frac{1}{\sqrt{\kappa^{2} \cos ^{2} \theta+k_{z, e}^{2}}}\left(\begin{array}{c} 
\pm k_{z, e} \\
0 \\
-\kappa \cos \theta
\end{array}\right), \\
\hat{d}_{o, \pm}=\frac{1}{\sqrt{\kappa^{2} \cos ^{2} \theta+k_{z, o}^{2}}}\left(\begin{array}{c} 
\pm k_{z, o} \\
0 \\
-\kappa \cos \theta
\end{array}\right), \\
\hat{d}_{e \pm}=\frac{1}{k_{e} \sqrt{\kappa^{2} \cos ^{2} \theta+k_{z, e}^{2}}}\left(\begin{array}{c}
\kappa^{2} \sin \theta \cos \theta \\
-k_{z, e}^{2}-\kappa^{2} \cos { }^{2} \theta \\
\pm k_{z, e} \kappa \sin \theta
\end{array}\right),
\end{gathered}
$$


where $k_{o}=\sqrt{\kappa^{2}+k_{z, o}^{2}}$ and $k_{e}=\sqrt{\kappa^{2}+k_{z, e}^{2}}$. Following the mode expansion denoted in Section 2.3 the equations determining the fields launching from source plane can be gotten by applying (13) and (14) under current situation; the equality of coefficients in front of $\delta\left(z-z_{0}\right)$ and $\delta^{\prime}(z-$ $z_{0}$ ) provides the equations determining these fields and constraints about the local field $\left(B_{x}, B_{y}\right)=4 \pi \mu\left(M_{x}, M_{y}\right)$ and $\left(E_{x}, E_{y}\right)=0$. We summarize them from (13) and (14), respectively, for $x, y$, and $z$ directions:

$$
\begin{aligned}
& \frac{\sqrt{\kappa^{2} \cos ^{2} \theta+k_{z, o}^{2}}}{k_{o}}\left(B_{o, g,-}-B_{o, g,+}\right) \\
& =-i 4 \pi \tilde{\omega} \mu P_{x}+i 4 \pi \mu \kappa \sin \theta M_{z} \text {, } \\
& \frac{\kappa^{2} \sin \theta \cos \theta}{k_{o} \sqrt{\kappa^{2} \cos ^{2} \theta+k_{z, o}^{2}}}\left(B_{o, g,-}-B_{o, g,+}\right) \\
& +\frac{k_{z, e}}{\sqrt{\kappa^{2} \cos ^{2} \theta+k_{z, e}^{2}}}\left(B_{e, g,+}+B_{e, g,-}\right) \\
& =-i 4 \pi \tilde{\omega} \mu P_{y}-i 4 \pi \mu \kappa \cos \theta M_{z} \text {, } \\
& E_{z}=-\frac{4 \pi}{\varepsilon_{o}} P_{z}, \\
& \frac{\sqrt{\kappa^{2} \cos ^{2} \theta+k_{z, e}^{2}}}{\widetilde{\omega} \mu \varepsilon_{e}}\left(B_{e, g,+}-B_{e, g,-}\right) \\
& +i E_{z} \kappa \sin \theta-i \tilde{\omega} B_{x}=0, \\
& \frac{k_{o}}{\widetilde{\omega} \mu \varepsilon_{o}} \frac{k_{z, o}}{\sqrt{k_{z, o}^{2}+\kappa^{2} \cos ^{2} \theta}}\left(B_{o, g,+}+B_{o, g,-}\right) \\
& +\frac{1}{\widetilde{\omega} \mu \varepsilon_{o}} \frac{\kappa^{2} \sin \theta \cos \theta}{\sqrt{\kappa^{2} \cos ^{2} \theta+k_{z, e}^{2}}}\left(B_{e, g,+}-B_{e, g,-}\right) \\
& -i E_{z} \kappa \cos \theta-i \widetilde{\omega} B_{y}=0, \\
& B_{z}=0 .
\end{aligned}
$$

After solving (28), the fields launching from the source plane are determined:

$$
\begin{gathered}
B_{o, g,+}=-O_{1} P_{z}+O_{2} P_{x}+O_{3} M_{y}-O_{4} M_{x}-O_{5} M_{z}, \\
B_{o, g,-}=-O_{1} P_{z}-O_{2} P_{x}+O_{3} M_{y}-O_{4} M_{x}+O_{5} M_{z}, \\
B_{e, g,+}=-\bar{O}_{1} P_{y}+\bar{O}_{2} P_{x}+\bar{O}_{3} P_{z}+\bar{O}_{4} M_{x}-\bar{O}_{5} M_{z}, \\
B_{e, g,-}=-\bar{O}_{1} P_{y}+\bar{O}_{2} P_{x}-\bar{O}_{3} P_{z}-\bar{O}_{4} M_{x}-\bar{O}_{5} M_{z},
\end{gathered}
$$

where the coefficient $R$ in (30) is given as $R=$ $\sqrt{k_{z, o}^{2}+\kappa^{2} \cos ^{2} \theta} / \sqrt{k_{z, e}^{2}+\kappa^{2} \cos ^{2} \theta}$. The fields in the regions outside of the anisotropic medium can be determined by 
solving boundary equations, and they can be written for $z=0$ and $z=h$, respectively, as

$$
\begin{aligned}
& \frac{-\kappa^{2} \sin \theta \cos \theta}{k_{o} \sqrt{\kappa^{2} \cos ^{2} \theta+k_{z, o}^{2}}}\left(B_{o, b,+}+B_{o, b,-}+B_{o, g,-} e^{i k_{z, o} z_{0}}\right) \\
& +\frac{k_{z, e}}{\sqrt{\kappa^{2} \cos ^{2} \theta+k_{z, e}^{2}}}\left(B_{e, b,+}-B_{e, b,-}-B_{e, g,-} e^{i k_{z, e} z_{0}}\right) \\
& =\frac{-B_{s,-} k_{z, \mathrm{I}}}{\widetilde{\omega}} \cos \theta+B_{p,-} \sin \theta, \\
& \frac{\sqrt{\kappa^{2} \cos ^{2} \theta+k_{z, o}^{2}}}{k_{o}}\left(B_{o, b,+}+B_{o, b,-}+B_{o, g,-} e^{i k_{z, o} z_{0}}\right) \\
& =\frac{-B_{s,-} k_{z, \mathrm{I}}}{\widetilde{\omega}} \sin \theta-B_{p,-} \cos \theta \\
& \left(B_{o, b,+}-B_{o, b,-}-B_{o, g,-} e^{i k_{z, o} z_{0}}\right) \frac{k_{o} k_{z, o}}{\tilde{\omega} \mu \varepsilon_{o} \sqrt{\kappa^{2} \cos ^{2} \theta+k_{z, o}^{2}}} \\
& +\left(B_{e, b,+}+B_{e, b,-}+B_{e, g,-} e^{i k_{z, e} z_{0}}\right) \frac{\kappa^{2} \sin \theta \cos \theta}{\tilde{\omega} \mu \varepsilon_{o} \sqrt{\kappa^{2} \cos ^{2} \theta+k_{z, e}^{2}}} \\
& =B_{s,-} \sin \theta+B_{p,-} \frac{k_{z, \mathrm{I}}}{\widetilde{\omega}} \cos \theta, \\
& \left(B_{e, b,+}+B_{e, b,-}+B_{e, g,-} e^{i k_{z, e} z_{0}}\right) \frac{\left(-k_{z, e}^{2}-\kappa^{2} \cos ^{2} \theta\right)}{\tilde{\omega} \mu \varepsilon_{e} \sqrt{\kappa^{2} \cos ^{2} \theta+k_{z, e}^{2}}} \\
& =-B_{s,-} \cos \theta+B_{p,-} \frac{k_{z, \mathrm{I}}}{\widetilde{\omega}} \sin \theta, \\
& \left(B_{o, b,+} e^{i k_{z, o} h}+B_{o, g,+} e^{i k_{z, o}\left(h-z_{0}\right)}+B_{o, b,-} e^{-i k_{z, o} h}\right) \\
& \times \frac{-\kappa^{2} \sin \theta \cos \theta}{k_{o} \sqrt{\kappa^{2} \cos ^{2} \theta+k_{z, o}^{2}}}
\end{aligned}
$$

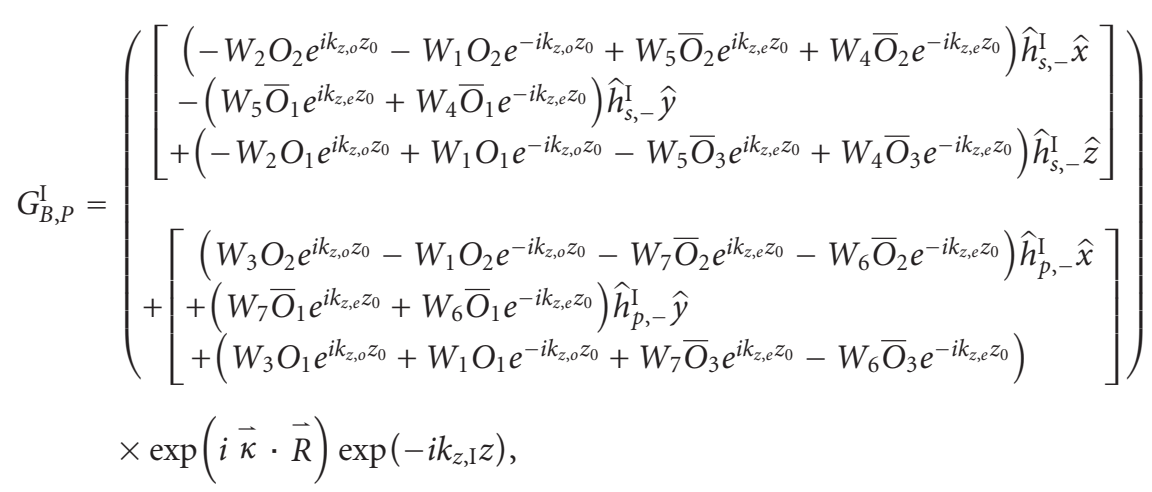

From these equations, the origin of mode conversion is obviously accounted by the equations with both $o$ - and $e$ mode. After solving the fields in regions I and III and linking to the sources with the help of (29), we can get Green functions for these regions: 


$$
\begin{aligned}
& G_{E, P}^{\mathrm{I}}=\left(\begin{array}{l}
{\left[\begin{array}{l}
\left(-W_{2} O_{2} e^{i k_{z, o} z_{0}}-W_{1} O_{2} e^{-i k_{z, o} z_{0}}+W_{5} \bar{O}_{2} e^{i k_{z, e} z_{0}}+W_{4} \bar{O}_{2} e^{-i k_{z, e} z_{0}}\right) \hat{e}_{s,-}^{\mathrm{I}} \hat{x} \\
-\left(W_{5} \bar{O}_{1} e^{i k_{z, e} z_{0}}+W_{4} \bar{O}_{1} e^{-i k_{z, e} z_{0}}\right) \hat{e}_{s,-}^{\mathrm{I}} \hat{y} \\
+\left(-W_{2} O_{1} e^{i k_{z, o} z_{0}}+W_{1} O_{1} e^{-i k_{z, 0} z_{0}}-W_{5} \bar{O}_{3} e^{i k_{z, e} z_{0}}+W_{4} \bar{O}_{3} e^{-i k_{z, e} z_{0}}\right) \hat{e}_{s,-}^{\mathrm{I}} \hat{z}
\end{array}\right]} \\
\left.+\begin{array}{l}
\left(W_{3} \mathrm{O}_{2} e^{i k_{z, o} z_{0}}-W_{1} O_{2} e^{-i k_{z, o} z_{0}}-W_{7} \bar{O}_{2} e^{i k_{z, e} z_{0}}-W_{6} \bar{O}_{2} e^{-i k_{z, e} z_{0}}\right) \hat{e}_{p,-}^{\mathrm{I}} \hat{x} \\
+\left(W_{7} \bar{O}_{1} e^{i k_{z, e} z_{0}}+W_{6} \bar{O}_{1} e^{-i k_{z, e} z_{0}}\right) \hat{e}_{p,-}^{\mathrm{I}} \hat{y} \\
+\left(W_{3} O_{1} e^{i k_{z, o} z_{0}}+W_{1} O_{1} e^{-i k_{z, o} z_{0}}+W_{7} \bar{O}_{3} e^{i k_{z, e} z_{0}}-W_{6} \bar{O}_{3} e^{-i k_{z, e} z_{0}}\right) \hat{e}_{p,-}^{\mathrm{I}} \hat{z}
\end{array}\right]
\end{array}\right) \\
& \times \exp (i \vec{\kappa} \cdot \vec{R}) \exp \left(-i k_{z, \mathrm{I}} z\right), \\
& G_{B, M}^{\mathrm{I}}=\left(\begin{array}{l}
{\left[\begin{array}{l}
\left(-W_{2} O_{4} e^{i k_{z, o} z_{0}}+W_{1} O_{4} e^{-i k_{z, o} z_{0}}-W_{5} \bar{O}_{4} e^{i k_{z, e} z_{0}}+W_{4} \bar{O}_{4} e^{-i k_{z, e} z_{0}}\right) \hat{h}_{s,-}^{\mathrm{I}} \hat{x} \\
+\left(W_{2} O_{3} e^{i k_{z, o} z_{0}}-W_{1} O_{3} e^{-i k_{z, o} z_{0}}\right) \hat{h}_{s,-}^{\mathrm{I}} \hat{y}^{-W_{2}} \\
+\left(W_{2} O_{5} e^{i k_{z, o} z_{0}}+W_{1} O_{5} e^{-i k_{z, o} z_{0}}-W_{5} \bar{O}_{5} e^{i k_{z, e} z_{0}}-W_{4} \bar{O}_{5} e^{-i k_{z, e} z_{0}}\right) \hat{h}_{s,-}^{\mathrm{I}} \hat{z}
\end{array}\right]} \\
+\left[\begin{array}{l}
\left(W_{3} O_{4} e^{i k_{z, o} z_{0}}+W_{1} O_{4} e^{-i k_{z, o} z_{0}}+W_{7} \bar{O}_{4} e^{i k_{z, e} z_{0}}-W_{6} \bar{O}_{4} e^{-i k_{z, e} z_{0}}\right) \hat{h}_{p,-}^{\mathrm{I}} \hat{x} \\
-\left(W_{3} O_{3} e^{i k_{z, o} z_{0}} M_{y}+W_{1} O_{3} e^{-i k_{z, o} z_{0}}\right) \hat{h}_{p,-}^{\mathrm{I}} \hat{y} \\
+\left(-W_{3} O_{5} e^{i k_{z, o} z_{0}}+W_{1} O_{5} e^{-i k_{z, o} z_{0}}+W_{7} \bar{O}_{5} e^{i k_{z, e} z_{0}}+W_{6} \bar{O}_{5} e^{-i k_{z, e} z_{0}}\right) \hat{h}_{p,-}^{\mathrm{I}} \hat{z}
\end{array}\right]
\end{array}\right) \\
& \times \exp (i \vec{\kappa} \cdot \vec{R}) \exp \left(-i k_{z, \mathrm{I}} z\right) \text {, } \\
& G_{E, M}^{\mathrm{I}}=\left(\begin{array}{l}
{\left[\begin{array}{l}
\left(-W_{2} O_{4} e^{i k_{z, 0} z_{0}}+W_{1} O_{4} e^{-i k_{z, 0} z_{0}}-W_{5} \bar{O}_{4} e^{i k_{z, e} z_{0}}+W_{4} \bar{O}_{4} e^{-i k_{z, e} z_{0}}\right) \hat{e}_{s,-}^{\mathrm{I}} \hat{x} \\
+\left(W_{2} O_{3} e^{i k_{z, o} z_{0}}-W_{1} O_{3} e^{-i k_{z, o} z_{0}}\right) \hat{e}_{s,-}^{\mathrm{I}} \hat{y} \\
+\left(W_{2} O_{5} e^{i k_{z, o} z_{0}}+W_{1} O_{5} e^{-i k_{z, 0} z_{0}}-W_{5} \bar{O}_{5} e^{i k_{z, e} z_{0}}-W_{4} \bar{O}_{5} e^{-i k_{z, e} z_{0}}\right) \hat{e}_{s,-}^{\mathrm{I}} \hat{z}
\end{array}\right]} \\
+\left[\begin{array}{l}
\left(W_{3} O_{4} e^{i k_{z, o} z_{0}}+W_{1} O_{4} e^{-i k_{z, o} z_{0}}+W_{7} \bar{O}_{4} e^{i k_{z, e} z_{0}}-W_{6} \bar{O}_{4} e^{-i k_{z, e} z_{0}}\right) \hat{e}_{p,-}^{\mathrm{I}} \hat{x} \\
-\left(W_{3} O_{3} e^{i k_{z, o} z_{0}} M_{y}+W_{1} O_{3} e^{-i k_{z, o} z_{0}}\right) \hat{e}_{p,-}^{\mathrm{I}} \hat{y} \\
+\left(-W_{3} O_{5} e^{i k_{z, 0} z_{0}}+W_{1} O_{5} e^{-i k_{z, o} z_{0}}+W_{7} \bar{O}_{5} e^{i k_{z, e} z_{0}}+W_{6} \bar{O}_{5} e^{-i k_{z, e} z_{0}}\right) \hat{e}_{p,-}^{\mathrm{I}} \hat{z}
\end{array}\right]
\end{array}\right) \\
& \times \exp (i \vec{\kappa} \cdot \vec{R}) \exp \left(-i k_{z, \mathrm{I}} z\right) \text {, } \\
& \left.G_{B, P}^{\mathrm{III}}=\left(\begin{array}{l}
{\left[\begin{array}{l}
\left(-W_{1} O_{2} e^{-i k_{z, o}\left(h-z_{0}\right)}-W_{2} \mathrm{O}_{2} e^{i k_{z, o}\left(h-z_{0}\right)}+W_{4} \bar{O}_{2} e^{-i k_{z, e}\left(h-z_{0}\right)}+W_{5} \bar{O}_{2} e^{i k_{z, e}\left(h-z_{0}\right)}\right) \hat{h}_{s,+}^{\mathrm{III}} \hat{x} \\
-\left(W_{4} \bar{O}_{1} e^{-i k_{z, e}\left(h-z_{0}\right)}+W_{5} \bar{O}_{1} e^{i k_{z, e}\left(h-z_{0}\right)}\right) \hat{h}_{s,+}^{\mathrm{III}} \hat{y} \\
+\left(-W_{1} O_{1} e^{-i k_{z, o}\left(h-z_{0}\right)}+W_{2} O_{1} e^{i k_{z, o}\left(h-z_{0}\right)}-W_{4} \bar{O}_{3} e^{-i k_{z, e}\left(h-z_{0}\right)}+W_{5} \bar{O}_{3} e^{i k_{z, e}\left(h-z_{0}\right)}\right) \hat{h}_{s,+}^{\mathrm{III}} \hat{z}
\end{array}\right.}
\end{array}\right]\right) \\
& \times \exp (i \vec{\kappa} \cdot \vec{R}) \exp \left(i k_{z, \mathrm{III}}(z-h)\right),
\end{aligned}
$$




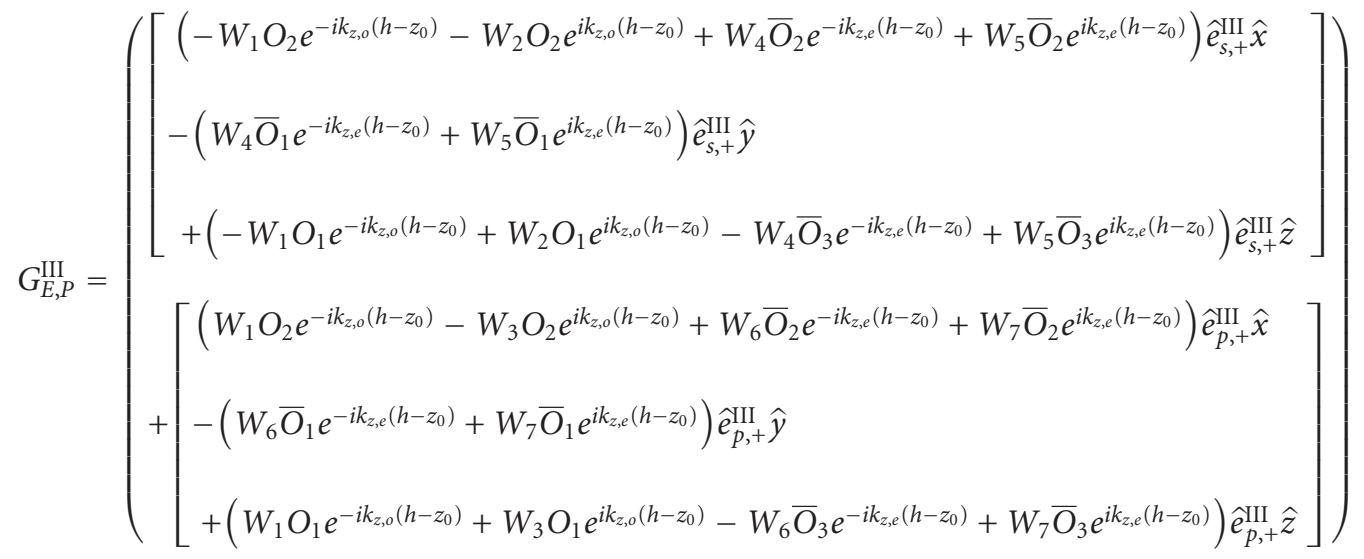

$$
\begin{aligned}
& \times \exp (i \vec{\kappa} \cdot \vec{R}) \exp \left(i k_{z, \mathrm{III}}(z-h)\right) \\
& G_{B, M}^{\mathrm{III}}=\left(\begin{array}{l}
{\left[\begin{array}{l}
\left(-W_{1} O_{4} e^{-i k_{z, o}\left(h-z_{0}\right)}+W_{2} O_{4} e^{i k_{z, o}\left(h-z_{0}\right)}-W_{4} \bar{O}_{4} e^{-i k_{z, e}\left(h-z_{0}\right)}+W_{5} \bar{O}_{4} e^{i k_{z, e}\left(h-z_{0}\right)}\right) \hat{h}_{s,+}^{\mathrm{III}} \hat{x} \\
+\left(W_{1} O_{3} e^{-i k_{z, o}\left(h-z_{0}\right)}-W_{2} O_{3} e^{i k_{z, o}\left(h-z_{0}\right)}\right) \hat{h}_{s,+}^{\mathrm{III}} \hat{y} \\
+\left(-W_{4} \bar{O}_{5} e^{-i k_{z, e}\left(h-z_{0}\right)}+W_{2} O_{5} e^{i k_{z, o}\left(h-z_{0}\right)} e^{-i k_{z} h}+W_{1} O_{5} e^{-i k_{z, o}\left(h-z_{0}\right)}-W_{5} \bar{O}_{5} e^{i k_{z, e}\left(h-z_{0}\right)}\right) \hat{h}_{s,+}^{\mathrm{III}} \hat{z}
\end{array}\right]} \\
+\left[\begin{array}{l}
\left(W_{1} O_{4} e^{-i k_{z, o}\left(h-z_{0}\right)}+W_{3} O_{4} e^{i k_{z, o}\left(h-z_{0}\right)}-W_{6} \bar{O}_{4} e^{-i k_{z, e}\left(h-z_{0}\right)}+W_{7} \bar{O}_{4} e^{i k_{z, e}\left(h-z_{0}\right)}\right) \hat{h}_{p,+}^{\mathrm{III}} \hat{x} \\
-\left(W_{1} O_{3} e^{-i k_{z, o}\left(h-z_{0}\right)}+W_{3} O_{3} e^{i k_{z, o}\left(h-z_{0}\right)}\right) \hat{h}_{p,+}^{\mathrm{III}} \hat{y} \\
+\left(-W_{1} O_{5} e^{-i k_{z, o}\left(h-z_{0}\right)}+W_{3} O_{5} e^{i k_{z, o}\left(h-z_{0}\right)}-W_{6} \bar{O}_{5} e^{-i k_{z, e}\left(h-z_{0}\right)}-W_{7} \bar{O}_{5} e^{i k_{z, e}\left(h-z_{0}\right)}\right) \hat{h}_{p,+}^{\mathrm{III}} \hat{z}
\end{array}\right]
\end{array}\right) \\
& \times \exp (i \vec{\kappa} \cdot \vec{R}) \exp \left(i k_{z, \mathrm{III}}(z-h)\right)
\end{aligned}
$$

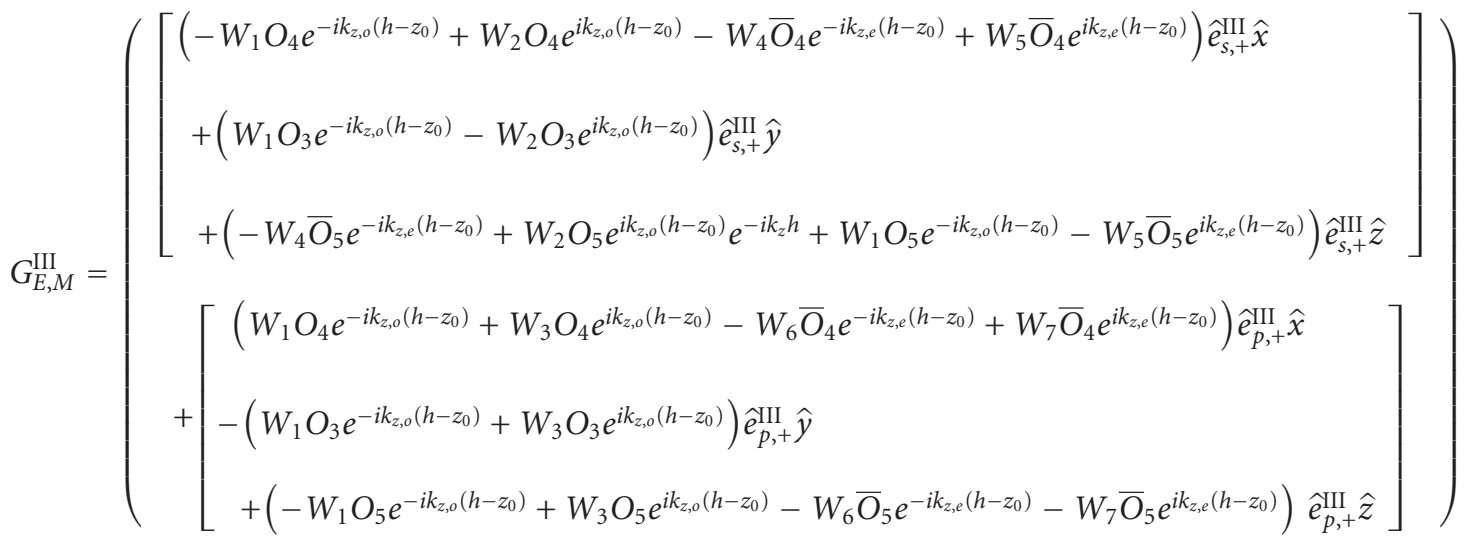

$$
\begin{aligned}
& \times \exp (i \vec{\kappa} \cdot \vec{R}) \exp \left(i k_{z, \text { III }}(z-h)\right) .
\end{aligned}
$$

The green functions in (32) describe the relation between the fields generated outside of the uniaxial crystal and the source embedded in uniaxial crystal. (The parameters $\left(W_{i}, i=\right.$ $1,2, \ldots, 7)$ in (32) will be given in Appendix A.)
3.2. Sources in Biaxial Crystal. In the subsection, the Green functions linking the fields in region I and III and sources embedded in biaxial crystal in region II are given. The relative permittivity tensor now has the form $\operatorname{diag}\left(\varepsilon_{1}, \varepsilon_{2}, \varepsilon_{3}\right)$ with 
$\varepsilon_{1} \neq \varepsilon_{2} \neq \varepsilon_{3}$. Thus, the equation determining the dispersions of eigenmodes now becomes

$$
\begin{aligned}
\tilde{\omega}^{4}- & \tilde{\omega}^{2}\left(\frac{k_{x}^{2}+k_{y}^{2}}{\varepsilon_{3} \mu}+\frac{k_{x}^{2}+k_{z}^{2}}{\varepsilon_{2} \mu}+\frac{k_{z}^{2}+k_{y}^{2}}{\varepsilon_{1} \mu}\right) \\
& +\left(\frac{k_{x}^{2}}{\varepsilon_{2} \varepsilon_{3} \mu^{2}}+\frac{k_{y}^{2}}{\varepsilon_{1} \varepsilon_{3} \mu^{2}}+\frac{k_{z}^{2}}{\varepsilon_{1} \varepsilon_{3} \mu^{2}}\right)\left(k_{x}^{2}+k_{z}^{2}+k_{y}^{2}\right)=0 .
\end{aligned}
$$

The dispersion can be gotten as followes: $k_{z, 1}^{2}=(-b+$ $\left.\sqrt{b^{2}-4 a c}\right) / 2 a$ and $k_{z, 2}^{2}=\left(-b-\sqrt{b^{2}-4 a c}\right) / 2 a$ (each of them denotes two modes propagating (or decaying) along positive and negative $z$ direction), where $a, b$, and $c$ are, respectively, the coefficients in front of the fourth-order, second-order and zeroth-order terms of $k_{z}$, and they have the following concrete forms:

$$
\begin{aligned}
a= & \frac{1}{\varepsilon_{1} \varepsilon_{3} \mu}, \quad b=\frac{k_{x}^{2}}{\varepsilon_{2} \varepsilon_{3} \mu}+\frac{k_{y}^{2}}{\varepsilon_{1} \varepsilon_{3} \mu}-\frac{\widetilde{\omega}^{2}}{\varepsilon_{2} \mu}-\frac{\widetilde{\omega}^{2}}{\varepsilon_{1} \mu} \\
c= & \left(k_{x}^{2}+k_{y}^{2}\right)\left(\frac{k_{x}^{2}}{\varepsilon_{2} \varepsilon_{3} \mu}+\frac{k_{y}^{2}}{\varepsilon_{1} \varepsilon_{3} \mu}\right) \\
& +k_{x}^{2}\left(\frac{1}{\varepsilon_{3} \mu}+\frac{\widetilde{\omega}^{2}}{\varepsilon_{2} \mu}\right)+k_{y}^{2}\left(\frac{1}{\varepsilon_{3} \mu}+\frac{\widetilde{\omega}^{2}}{\varepsilon_{1} \mu}\right)+\widetilde{\omega}^{4}
\end{aligned}
$$

The corresponding unit vectors of electric fields of eigenmodes can be solved as [23]

$$
\hat{e}_{i, \pm}=\left(X_{i}^{2}+1+Z_{i}^{2}\right)^{-1 / 2}\left(X_{i}, 1, \pm Z_{i}\right)
$$

where

$$
\begin{aligned}
& X_{i}=\frac{\kappa^{2} \cos \theta \sin \theta\left(\kappa^{2}+k_{z, i}^{2}-\widetilde{\omega}^{2} \varepsilon_{3} \mu\right)}{\left(\widetilde{\omega}^{2} \varepsilon_{1} \mu-k_{z, i}^{2}-\kappa^{2} \sin ^{2} \theta\right)\left(\widetilde{\omega}^{2} \varepsilon_{3} \mu-\kappa^{2}\right)-\kappa^{2} \cos ^{2} \theta k_{z, i}^{2}} \\
& Z_{i}=\frac{k_{z, i} \kappa \sin \theta\left(\kappa^{2}+k_{z, i}^{2}-\widetilde{\omega}^{2} \varepsilon_{1} \mu\right)}{\left(\widetilde{\omega}^{2} \varepsilon_{1} \mu-\kappa^{2} \sin ^{2} \theta-k_{z, i}^{2}\right)\left(\widetilde{\omega}^{2} \varepsilon_{3} \mu-\kappa^{2}\right)-\kappa^{2} \cos ^{2} \theta k_{z, i}^{2}} .
\end{aligned}
$$

$$
\begin{aligned}
& \hat{d}_{i, \pm}=\left(\varepsilon_{1}^{2} X_{i}^{2}+\varepsilon_{2}^{2}+\varepsilon_{3}^{2} Z_{i}^{2}\right)^{-1 / 2}\left(\varepsilon_{1} X_{i}, \varepsilon_{2}, \pm \varepsilon_{3} Z_{i}\right), \\
& \hat{h}_{i, \pm}=\frac{1}{N_{i}}\left( \pm\left(\kappa \sin \theta Z_{i}-k_{z, i}\right), \pm\left(k_{z, i} X_{i}-Z_{i} \kappa \cos \theta\right),\left(\kappa \cos \theta-\kappa \sin \theta X_{i}\right)\right),
\end{aligned}
$$

$$
\text { where } N_{i}=\sqrt{\left(\kappa \sin \theta Z_{i}-k_{z, i}\right)^{2}+\left(k_{z, i} X_{i}-Z_{i} \kappa \cos \theta\right)^{2}+\left(\kappa \cos \theta-\kappa \sin \theta X_{i}\right)^{2}} \text {. }
$$

In (36) $k_{z, i}$ is a positive square root from $k_{z, i}^{2}$ so that it denotes the propagating or decaying wave along positive $z$ direction. Thus, the unit vectors of electric displacement vector and magnetic field can be gotten as
Then we can determine the fields launching from the source plane with the help of these unit vectors defined. The general equations (13) and (14) can be mapped to concrete forms with each equation gotten giving the field equality in different directions. The relations, $\left(B_{x}, B_{y}\right)=4 \pi \mu\left(M_{x}, M_{y}\right)$ and $\left(E_{x}, E_{y}\right)=0$, are found again by following the fact of vanishing of the coefficients in front of $\delta^{\prime}$ function. The equations determining the fields launching from the source plane are:

$$
\begin{aligned}
& \frac{k_{1}}{\tilde{\omega} \mu\left(\varepsilon_{1}^{2} X_{1}^{2}+\varepsilon_{2}^{2}+\varepsilon_{3}^{2} Z_{1}^{2}\right)^{1 / 2}}\left[B_{1, g,-}-B_{1, g,+}\right] \\
& +\frac{k_{2}}{\tilde{\omega} \mu\left(\varepsilon_{1}^{2} X_{2}^{2}+\varepsilon_{2}^{2}+\varepsilon_{3}^{2} Z_{2}^{2}\right)^{1 / 2}}\left[B_{2, g,-}-B_{2, g,+}\right] \\
& +i E_{z} \kappa \sin \theta=i \tilde{\omega} B_{x}, \\
& \frac{X_{1} k_{1}}{\tilde{\omega} \mu\left(\varepsilon_{1}^{2} X_{1}^{2}+\varepsilon_{2}^{2}+\varepsilon_{3}^{2} Z_{1}^{2}\right)^{1 / 2}}\left[B_{1, g,-}-B_{1, g,+}\right] \\
& +\frac{X_{2} k_{2}}{\tilde{\omega} \mu\left(\varepsilon_{1}^{2} X_{2}^{2}+\varepsilon_{2}^{2}+\varepsilon_{3}^{2} Z_{2}^{2}\right)^{1 / 2}}\left[B_{2, g,-}-B_{2, g,+}\right] \\
& -i E_{z} \kappa \cos \theta=i \tilde{\omega} B_{y},
\end{aligned}
$$

$$
\begin{gathered}
B_{z}=0 \\
-\frac{\left(k_{z, 1} X_{1}-Z_{1} \kappa \cos \theta\right)}{N_{1}}\left(B_{1, g,+}+B_{1, g,-}\right) \\
-\frac{\left(k_{z, 2} X_{2}-Z_{2} \kappa \cos \theta\right)}{N_{2}}\left(B_{2, g,+}+B_{2, g,-}\right) \\
=-i 4 \pi \tilde{\omega} \mu P_{x}+i 4 \pi \mu \kappa \sin \theta M_{z}, \\
\frac{\left(\kappa \sin \theta Z_{1}-k_{z, 1}\right)}{N_{1}}\left(B_{1, g,+}+B_{1, g,-}\right) \\
+\frac{\left(\kappa \sin \theta Z_{2}-k_{z, 2}\right)}{N_{2}}\left(B_{2, g,+}+B_{2, g,-}\right) \\
=-i 4 \pi \tilde{\omega} \mu P_{y}-i 4 \pi \mu \kappa \cos \theta M_{z}, \\
E_{z}=-\frac{4 \pi}{\varepsilon_{3}} P_{z},
\end{gathered}
$$

where $k_{1}=\sqrt{\kappa^{2}+k_{z, 1}^{2}}$ and $k_{2}=\sqrt{\kappa^{2}+k_{z, 2}^{2}}$. The solutions of (35) and (36) give the relations between the fields launching 
from the source plane and the sources as

$$
\begin{gathered}
B_{1, g,-}=\frac{1}{2}\left(V_{10} P_{x}+V_{11} P_{y}+V_{12} M_{z}+V_{1} M_{x}+V_{2} M_{y}+V_{3} P_{z}\right) \\
B_{1, g,+}=\frac{1}{2}\left(V_{10} P_{x}+V_{11} P_{y}+V_{12} M_{z}-V_{1} M_{x}-V_{2} M_{y}-V_{3} P_{z}\right) \\
B_{2, g,-}=\frac{1}{2}\left(V_{7} P_{x}+V_{8} P_{y}+V_{9} M_{z}+V_{4} M_{x}+V_{5} M_{y}+V_{6} P_{z}\right) \\
B_{2, g,+}=\frac{1}{2}\left(V_{7} P_{x}+V_{8} P_{y}+V_{9} M_{z}-V_{4} M_{x}-V_{5} M_{y}-V_{6} P_{z}\right) .
\end{gathered}
$$

The details of the definitions of parameters $V_{i}(i=1,2$, $\ldots, 12)$ in (39) are given in Appendix B. To determine the fields generated in regions I and III, the boundary equations should be solved, and we firstly write down their concrete forms from the continuity of the tangential fields for the interfaces at $z=0$ and $z=h$, respectively (we have used the fact that $k_{z}=k_{z, \mathrm{I}}=k_{z, \text { III }}$ in the following equations, when $\vec{\kappa}$ is the same),

$$
\begin{aligned}
& S_{1}\left(B_{1, b,+}-B_{1, b,-}-B_{1, g,-} e^{i k_{z, 1} z_{0}}\right) \\
& +S_{2}\left(B_{2, b,+}-B_{2, b,-}-B_{2, g,-} e^{i k_{z, 2} z_{0}}\right) \\
& =\frac{-B_{s,-} k_{z}}{\widetilde{\omega}} \cos \theta+B_{p,-} \sin \theta, \\
& S_{3}\left(B_{1, b,+}-B_{1, b,-}-B_{1, g,-} e^{i k_{z, 1} z_{0}}\right) \\
& +S_{4}\left(B_{2, b,+}-B_{2, b,-}-B_{2, g,-} e^{i k_{z, 2} z_{0}}\right) \\
& =\frac{-B_{s,-} k_{z}}{\widetilde{\omega}} \sin \theta-B_{p,-} \cos \theta, \\
& S_{5} X_{1}\left(B_{1, b,+}+B_{1, b,-}+B_{1, g,-} e^{i k_{z, 1} z_{0}}\right) \\
& +S_{6} X_{2}\left(B_{2, b,+}+B_{2, b,-}+B_{2, g,-} e^{i k_{z, 2} z_{0}}\right) \\
& =B_{s,-} \sin \theta+B_{p,-} \frac{k_{z}}{\widetilde{\omega}} \cos \theta, \\
& S_{5}\left(B_{1, b,+}+B_{1, b,-}+B_{1, g,-} e^{i k_{z, 1} z_{0}}\right) \\
& +S_{6}\left(B_{2, b,+}+B_{2, b,-}+B_{2, g,-} e^{i k_{z, 2} z_{0}}\right) \\
& =-B_{s,-} \cos \theta+B_{p,-} \frac{k_{z}}{\widetilde{\omega}} \sin \theta, \\
& S_{1}\left(B_{1, b,+} e^{i k_{z, 1} h}-B_{1, b,-} e^{-i k_{z, 1} h}+B_{1, g,+} e^{i k_{z, 1}\left(h-z_{0}\right)}\right) \\
& +S_{2}\left(B_{2, b,+} e^{i k_{z, 2} h}-B_{2, b,-} e^{-i k_{z, 2} h}+B_{2, g,+} e^{i k_{z, 2}\left(h-z_{0}\right)}\right) \\
& =\frac{k_{z} \cos \theta}{\widetilde{\omega}} B_{s,+} e^{i k_{z} h}+\sin \theta B_{p,+} e^{i k_{z} h} \text {, }
\end{aligned}
$$

$$
\begin{aligned}
& S_{3}(\left.B_{1, b,+} e^{i k_{z, 1} h}-B_{1, b,-} e^{-i k_{z, 1} h}+B_{1, g,+} e^{i k_{z, 1}\left(h-z_{0}\right)}\right) \\
&+S_{4}\left(B_{2, b,+} e^{i k_{z, 2} h}-B_{2, b,-} e^{-i k_{z, 2} h}+B_{2, g,+} e^{i k_{z, 2}\left(h-z_{0}\right)}\right) \\
&= \frac{k_{z} \sin \theta}{\widetilde{\omega}} B_{s,+} e^{i k_{z} h}-\cos \theta B_{p,+} e^{i k_{z} h}, \\
& S_{5} X_{1}\left(B_{1, b,+} e^{i k_{z, 1} h}+B_{1, b,-} e^{-i k_{z, 1} h}+B_{1, g,+} e^{i k_{z, 1}\left(h-z_{0}\right)}\right) \\
&+S_{6} X_{2}\left(B_{2, b,+} e^{i k_{z, 2} h}+B_{2, b,-} e^{-i k_{z, 2} h}+B_{2, g,+} e^{i k_{z, 2}\left(h-z_{0}\right)}\right) \\
&= \sin \theta B_{s,+} e^{i k_{z} h}-\frac{k_{z} \cos \theta}{\widetilde{\omega}} B_{p,+} e^{i k_{z} h} \\
& S_{5}(\left.B_{1, b,+} e^{i k_{z, 1} h}+B_{1, b,-} e^{-i k_{z, 1} h}+B_{1, g,+} e^{i k_{z, 1}\left(h-z_{0}\right)}\right) \\
&+S_{6}\left(B_{2, b,+} e^{i k_{z, 2} h}+B_{2, b,-} e^{-i k_{z, 2} h}+B_{2, g,+} e^{i k_{z, 2}\left(h-z_{0}\right)}\right) \\
&=-\cos \theta B_{s,+} e^{i k_{z} h}-\frac{k_{z} \sin \theta}{\widetilde{\omega}} B_{p,+} e^{i k_{z} h},
\end{aligned}
$$

where the parameters are given as

$$
\begin{gathered}
S_{1}=\frac{\kappa \sin \theta Z_{1}-k_{z, 1}}{N_{1}}, \quad S_{2}=\frac{\kappa \sin \theta Z_{2}-k_{z, 2}}{N_{2}}, \\
S_{3}=\frac{k_{z, 1} X_{1}-Z_{1} \kappa \cos \theta}{N_{1}}, \quad S_{4}=\frac{k_{z, 2} X_{2}-Z_{2} \kappa \cos \theta}{N_{2}}, \\
S_{5}=\frac{k_{1}}{\tilde{\omega} \mu \sqrt{\varepsilon_{1}^{2} X_{1}^{2}+\varepsilon_{2}^{2}+\varepsilon_{3}^{2} Z_{1}^{2}}}, \\
S_{6}=\frac{k_{2}}{\tilde{\omega} \mu \sqrt{\varepsilon_{1}^{2} X_{2}^{2}+\varepsilon_{2}^{2}+\varepsilon_{3}^{2} Z_{2}^{2}}} .
\end{gathered}
$$

The fields in regions I and III can be solved out from (40) and (41). Consequently, the Green functions relating them to the sources in biaxial crystal can be gotten with the help of (39) and the definitions given in (8):

$$
\begin{aligned}
& \left.G_{B, P}^{\mathrm{I}}=\frac{1}{2}\left(\begin{array}{c}
{\left[\begin{array}{l}
\left(U_{2} V_{10} e^{i k_{z, 1} z_{0}}-U_{1} V_{10} e^{-i k_{z, 1} z_{0}}+U_{5} V_{7} e^{i k_{z, 2} z_{0}}+U_{4} V_{7} e^{-i k_{z, 2} z_{0}}\right) \hat{h}_{s,-}^{\mathrm{I}} \hat{x} \\
+\left(U_{2} V_{11} e^{i k_{z, 1} z_{0}}-U_{1} V_{11} e^{-i k_{z, 1} z_{0}}+U_{5} V_{8} e^{i k_{z, 2} z_{0}}+U_{4} V_{8} e^{-i k_{z, 2} z_{0}}\right) \hat{h}_{s,-}^{\mathrm{I}} \hat{y} \\
+\left(U_{2} V_{3} e^{i k_{z, 1} z_{0}}+U_{1} V_{3} e^{-i k_{z, 1} z_{0}}+U_{5} V_{6} e^{i k_{z, 2} z_{0}}-U_{4} V_{6} e^{-i k_{z, 2} z_{0}}\right) \hat{h}_{s,-}^{\mathrm{I}} \hat{z}
\end{array}\right]} \\
+\left[\begin{array}{c}
\left(-U_{3} V_{10} e^{i k_{z, 1} z_{0}}-U_{1} V_{10} e^{-i k_{z, 1} z_{0}}-U_{7} V_{7} e^{i k_{z, 2} z_{0}}-U_{6} V_{7} e^{-i k_{z, 2} z_{0}}\right) \hat{h}_{p,-}^{\mathrm{I}} \hat{x} \\
+\left(-U_{3} V_{11} e^{i k_{z, 1} z_{0}}-U_{1} V_{11} e^{-i k_{z, 1} z_{0}}-U_{7} V_{8} e^{i k_{z, 2} z_{0}}-U_{6} V_{8} e^{-i k_{z, 2} z_{0}}\right) \\
+\left(-U_{3} V_{3} e^{i k_{z, 1} z_{0}}+U_{1} V_{3} e^{-i k_{z, 1} z_{0}}-U_{7} V_{6} e^{i k_{z, 2} z_{0}}+U_{6} V_{6} e^{-i k_{z, 2} z_{0}}\right)
\end{array}\right) \hat{h}_{p,-}^{\mathrm{I}} \hat{z}
\end{array}\right]\right) \\
& \times \exp (i \vec{\kappa} \cdot \vec{R}) \exp \left(-i k_{z, \mathrm{I}} z\right) \text {, }
\end{aligned}
$$




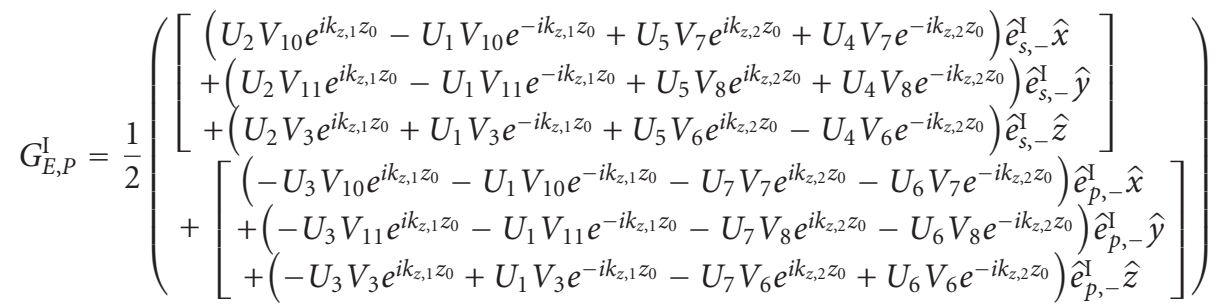

$$
\begin{aligned}
& \times \exp (i \vec{\kappa} \cdot \vec{R}) \exp \left(-i k_{z, \mathrm{I}} z\right),
\end{aligned}
$$

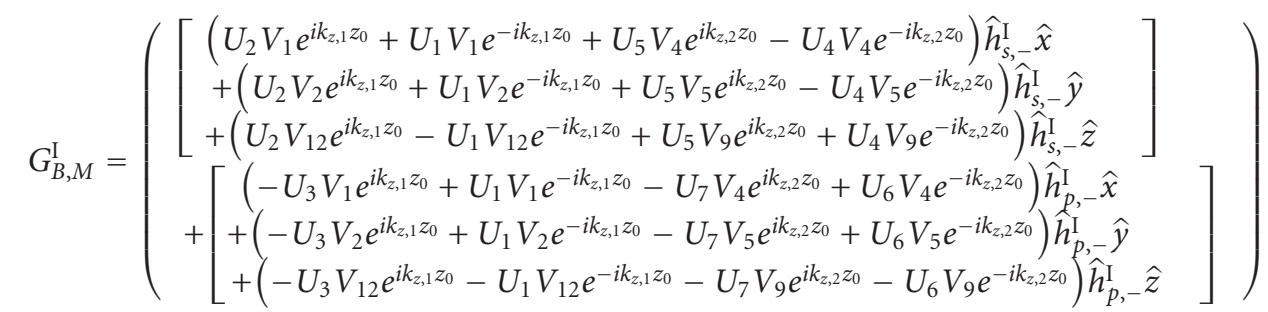

$$
\begin{aligned}
& \times \exp (i \vec{\kappa} \cdot \vec{R}) \exp \left(-i k_{z, \mathrm{I}} z\right),
\end{aligned}
$$

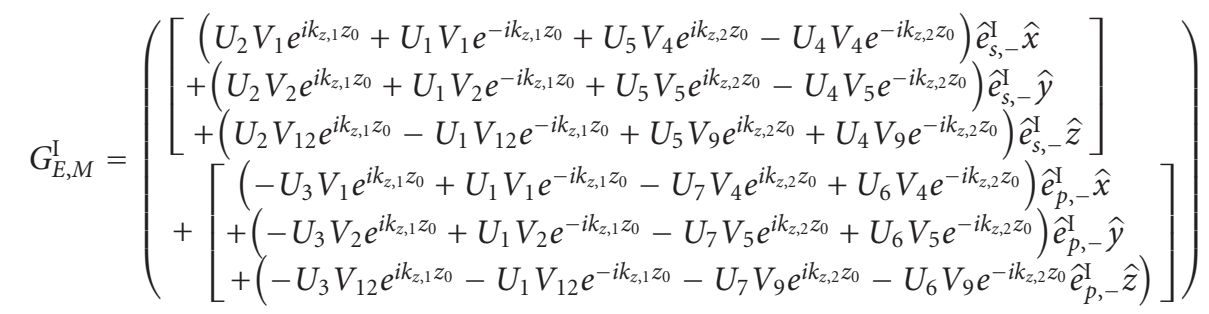

$$
\begin{aligned}
& \times \exp (i \vec{\kappa} \cdot \vec{R}) \exp \left(-i k_{z, \mathrm{I}} z\right), \\
& \left.G_{B, P}^{\mathrm{III}}=\frac{1}{2}\left(\begin{array}{c}
{\left[\begin{array}{l}
\left(U_{1} V_{10} e^{-i k_{z, 1}\left(h-z_{0}\right)}-U_{2} V_{10} e^{i k_{z, 1}\left(h-z_{0}\right)}+U_{4} V_{7} e^{-i k_{z, 2}\left(h-z_{0}\right)}+U_{5} V_{7} e^{i k_{z, 2}\left(h-z_{0}\right)}\right) \hat{h}_{s,+}^{\mathrm{III}} \hat{x} \\
+\left(U_{1} V_{11} e^{-i k_{z, 1}\left(h-z_{0}\right)}-U_{2} V_{11} e^{i k_{z, 1}\left(h-z_{0}\right)}+U_{4} V_{8} e^{-i k_{z, 2}\left(h-z_{0}\right)}+U_{5} V_{8} e^{i k_{z, 2}\left(h-z_{0}\right)}\right) \\
+\left(U_{1} V_{3} e^{-i k_{z, 1}\left(h-z_{0}\right)}+U_{2} V_{3} e^{i k_{z, 1}\left(h-z_{0}\right)}+U_{4} V_{6} e^{-i k_{z, 2}\left(h-z_{0}\right)}-U_{5} V_{6} e^{i k_{z, 2}\left(h-z_{0}\right)}\right) \hat{h}_{s,+}^{\mathrm{II}} \hat{z}
\end{array}\right]} \\
+\left[\begin{array}{l}
\left(-U_{1} V_{10} e^{-i k_{z, 1}\left(h-z_{0}\right)}-U_{3} V_{10} e^{i k_{z, 1}\left(h-z_{0}\right)}+U_{6} V_{7} e^{-i k_{z, 2}\left(h-z_{0}\right)}+U_{7} V_{7} e^{i k_{z, 2}\left(h-z_{0}\right)}\right) \\
+\left(-U_{1} V_{11} e^{-i k_{z, 1}\left(h-z_{0}\right)}-U_{3} V_{11} e^{i k_{z, 1}\left(h-z_{0}\right)}+U_{6} V_{8} e^{-i k_{z, 2}\left(h-z_{0}\right)}+U_{7} V_{8} e^{i k_{z, 2}\left(h-z_{0}\right)}\right) \\
+\left(-U_{1} V_{3} e^{-i k_{z, 1}\left(h-z_{0}\right)}+U_{3} V_{3} e^{i k_{z, 1}\left(h-z_{0}\right)}+U_{6} V_{6} e^{-i k_{z, 2}\left(h-z_{0}\right)}-U_{7} V_{6} e^{i k_{z, 2}\left(h-z_{0}\right)}\right)
\end{array}\right) \hat{h}_{p,+}^{\mathrm{III}} \hat{z}
\end{array}\right]\right) \\
& \times \exp (i \vec{\kappa} \cdot \vec{R}) \exp \left(i k_{z, \mathrm{III}}(z-h)\right) \text {, }
\end{aligned}
$$

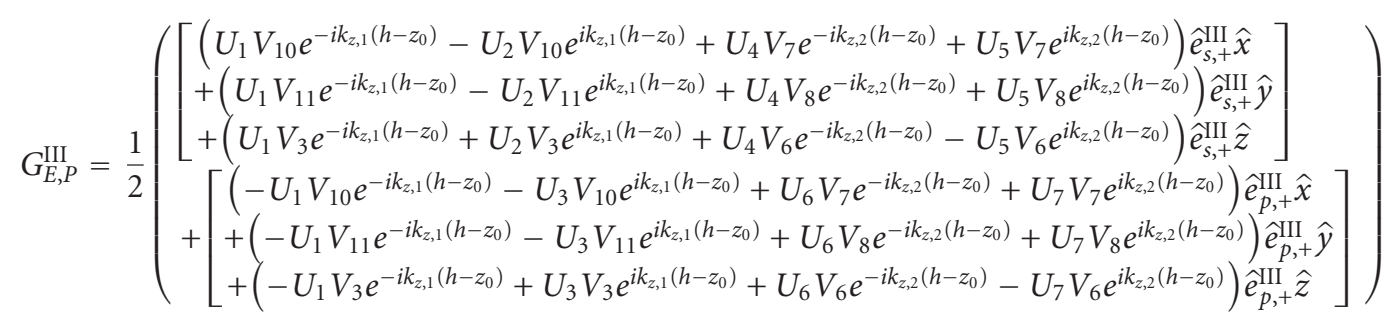

$$
\begin{aligned}
& \times \exp (i \vec{\kappa} \cdot \vec{R}) \exp \left(i k_{z, \mathrm{III}}(z-h)\right) \text {, }
\end{aligned}
$$

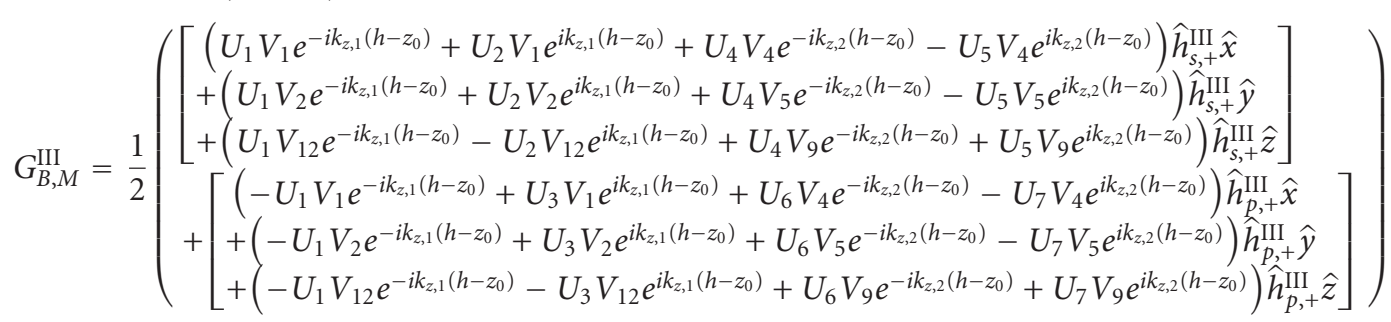

$$
\begin{aligned}
& \times \exp (i \vec{\kappa} \cdot \vec{R}) \exp \left(i k_{z, \mathrm{III}}(z-h)\right) \text {, }
\end{aligned}
$$




$$
\begin{aligned}
& G_{E, M}^{\mathrm{III}}=\frac{1}{2}\left(\begin{array}{c}
{\left[\begin{array}{c}
\left(U_{1} V_{1} e^{-i k_{z, 1}\left(h-z_{0}\right)}+U_{2} V_{1} e^{i k_{z, 1}\left(h-z_{0}\right)}+U_{4} V_{4} e^{-i k_{z, 2}\left(h-z_{0}\right)}-U_{5} V_{4} e^{i k_{z, 2}\left(h-z_{0}\right)}\right) \hat{e}_{s,+}^{\mathrm{III}} \hat{x} \\
+\left(U_{1} V_{2} e^{-i k_{z, 1}\left(h-z_{0}\right)}+U_{2} V_{2} e^{i k_{z, 1}\left(h-z_{0}\right)}+U_{4} V_{5} e^{-i k_{z, 2}\left(h-z_{0}\right)}-U_{5} V_{5} e^{i k_{z, 2}\left(h-z_{0}\right)}\right) \hat{e}_{s,+}^{\mathrm{III}} \hat{y} \\
+\left(U_{1} V_{12} e^{-i k_{z, 1}\left(h-z_{0}\right)}-U_{2} V_{12} e^{i k_{z, 1}\left(h-z_{0}\right)}+U_{4} V_{9} e^{-i k_{z, 2}\left(h-z_{0}\right)}+U_{5} V_{9} e^{i k_{z, 2}\left(h-z_{0}\right)}\right) \hat{e}_{s,+}^{\mathrm{III}} \hat{z}
\end{array}\right]} \\
+\left[\begin{array}{c}
\left(-U_{1} V_{1} e^{-i k_{z, 1}\left(h-z_{0}\right)}+U_{3} V_{1} e^{i k_{z, 1}\left(h-z_{0}\right)}+U_{6} V_{4} e^{-i k_{z, 2}\left(h-z_{0}\right)}-U_{7} V_{4} e^{i k_{z, 2}\left(h-z_{0}\right)}\right) \hat{e}_{p,+}^{\mathrm{III}} \hat{x} \\
+\left(-U_{1} V_{2} e^{-i k_{z, 1}\left(h-z_{0}\right)}+U_{3} V_{2} e^{i k_{z, 1}\left(h-z_{0}\right)}+U_{6} V_{5} e^{-i k_{z, 2}\left(h-z_{0}\right)}-U_{7} V_{5} e^{i k_{z, 2}\left(h-z_{0}\right)}\right) \hat{e}_{p,+}^{\mathrm{III}} \hat{y} \\
+\left(-U_{1} V_{12} e^{-i k_{z, 1}\left(h-z_{0}\right)}-U_{3} V_{12} e^{i k_{z, 1}\left(h-z_{0}\right)}+U_{6} V_{9} e^{-i k_{z, 2}\left(h-z_{0}\right)}+U_{7} V_{9} e^{i k_{z, 2}\left(h-z_{0}\right)}\right) \hat{e}_{p,+}^{\mathrm{III}} \hat{z}
\end{array}\right]
\end{array}\right) \\
& \times \exp (i \vec{\kappa} \cdot \vec{R}) \exp \left(i k_{z, \text { III }}(z-h)\right) \text {. }
\end{aligned}
$$

Green functions gotten in (42) give the relations between the fields generated in region I (or region III) and the sources embedded in biaxial crystal (region II). The definitions of parameters $\left(U_{i}, i=1,2, \ldots, 7\right)$ in (42) will be given in Appendix C.

\section{Conclusion Remark}

In summary, a new Green function formulism is developed by combining eigenmode expansion method and conventional formulism. The correction of the formulism is supported by recovering well-known results gotten by conventional formulism. The powerful eigenmode expansion method removes the difficulties brought by the interface between anisotropic media. Given to the advantage, the Green functions relating the fields generated outside of anisotropic medium and the sources inside were calculated and shown. They may have potential applications in studying the electromagnetic response and its spatial distribution of anisotropic metamaterial medium, which has well-defined effective medium tensor.

\section{Appendices}

\section{A. Definitions of Parameters $W_{i}$ in (32)}

The parameters $W_{i}$ come out, when one solves the equation groups (31). Thus, it can be related to the coefficients in front of the field amplitudes. In the solution processes, they firstly relate to parameters $I_{i}, i=1,2, \ldots, 6$, and $F_{i}, i=1,2, \ldots, 9$, as

$$
\begin{aligned}
& W_{1}=\left[\frac{I_{3} I_{5}-I_{2} I_{6}}{I_{1} I_{5}-I_{2} I_{4}} e^{i k_{z, o} h}\right], \\
& W_{2}=\left[\frac{F_{1} I_{5}-F_{4} I_{2}}{F_{7}\left(I_{1} I_{5}-I_{2} I_{4}\right)} e^{-i k_{z, 0} h}\right], \\
& W_{3}=\left[\frac{F_{1} I_{4}-F_{4} I_{1}}{F_{7}\left(I_{2} I_{4}-I_{1} I_{5}\right)} e^{-i k_{z, o} h}\right], \\
& W_{4}=\frac{I_{2}}{I_{1} I_{5}-I_{2} I_{4}} e^{i k_{z, o} h},
\end{aligned}
$$

$$
\begin{aligned}
& W_{5}=\frac{I_{5}}{I_{1} I_{5}-I_{2} I_{4}} e^{-i k_{z, o} h}, \\
& W_{6}=\frac{I_{1}}{I_{2} I_{4}-I_{1} I_{5}} e^{i k_{z, o} h}, \\
& W_{7}=\frac{I_{4}}{I_{2} I_{4}-I_{1} I_{5}} e^{-i k_{z, o} h} .
\end{aligned}
$$

The definitions of parameters $I_{i}, i=1,2, \ldots, 6$, are the following:

$$
\begin{aligned}
& I_{1}=F_{2}-\frac{F_{1} F_{8}}{F_{7}}, \\
& I_{2}=F_{3}-\frac{F_{1} F_{9}}{F_{7}}, \\
& I_{3}=\frac{F_{1} D_{6}}{F_{7} D_{5}}+\frac{D_{2}}{D_{5}}, \\
& I_{4}=F_{5}-\frac{F_{4} F_{8}}{F_{7}}, \\
& I_{5}=F_{6}-\frac{F_{4} F_{9}}{F_{7}}, I_{6}=\frac{F_{4} D_{6}}{F_{7} D_{5}}-\frac{D_{1}}{D_{5}} .
\end{aligned}
$$

The definitions of parameters $F_{i}, i=1,2, \ldots, 9$, are given as

$$
\begin{aligned}
& F_{1}=\frac{D_{2} D_{7}}{D_{5}}+D_{4}, \\
& F_{2}=D_{1} e^{-i k_{z, e} h}+\frac{D_{2} D_{6}}{D_{5}} e^{i k_{z, 0} h}, \\
& F_{3}=D_{3} e^{-i k_{z, e} h}-\frac{D_{2} D_{8}}{D_{5}} e^{i k_{z, o} h}, \\
& F_{4}=-\frac{D_{1} D_{7}}{D_{5}}-D_{3}, \\
& F_{5}=-D_{2} e^{i k_{z, e} h}-\frac{D_{1} D_{6}}{D_{5}} e^{i k_{z, o} h}, \\
& F_{6}=-D_{4} e^{i k_{z, e} h}+\frac{D_{1} D_{8}}{D_{5}} e^{i k_{z, 0} h},
\end{aligned}
$$




$$
\begin{aligned}
& F_{7}=D_{8}-\frac{D_{6} D_{7}}{D_{5}}, \\
& F_{8}=D_{5} e^{-i k_{z, o} h}-\frac{D_{6}^{2}}{D_{5}} e^{i k_{z, o} h}, \\
& F_{9}=-D_{7} e^{-i k_{z, o} h}+\frac{D_{6} D_{8}}{D_{5}} e^{i k_{z, o} h} .
\end{aligned}
$$

The parameters $D_{i}, i=1,2, \ldots, 8$, relate to the coefficients in (31) directly (since the media in region I and region III are the same, we used the fact that $k_{z}=k_{z, \mathrm{I}}=k_{z, \text { III }}$, when $\vec{\kappa}$ is the same):

$$
\begin{aligned}
& D_{1}=\frac{k_{z}}{2 A_{2} A_{3} \widetilde{\omega}}\left(A_{3} \cos \theta+A_{1} \sin \theta\right)+\frac{\tilde{\omega} \mu \varepsilon_{e} R}{2 k_{o} A_{3}} \cos \theta \\
& D_{2}=\frac{k_{z}}{2 A_{2} A_{3} \widetilde{\omega}}\left(A_{3} \cos \theta+A_{1} \sin \theta\right)-\frac{\tilde{\omega} \mu \varepsilon_{e} R}{2 k_{o} A_{3}} \cos \theta \\
& D_{3}=\frac{1}{2 A_{2} A_{3}}\left(A_{3} \sin \theta-A_{1} \cos \theta\right)+\frac{k_{z} \mu \varepsilon_{e} R}{2 k_{o} A_{3}} \sin \theta \\
& D_{4}=\frac{1}{2 A_{2} A_{3}}\left(A_{3} \sin \theta-A_{1} \cos \theta\right)-\frac{k_{z} \mu \varepsilon_{e} R}{2 k_{o} A_{3}} \sin \theta \\
& D_{5}=\frac{k_{z, e} \tilde{\omega} \mu \varepsilon_{e} \sqrt{\mu \varepsilon_{o}} R^{2}}{2 k_{z, o} k_{o} A_{2} A_{3}}\left(\frac{k_{o} \sin \theta}{\widetilde{\omega} \mu \varepsilon_{e} R} A_{3}-\frac{R \cos \theta}{\sqrt{\mu \varepsilon_{o}}} A_{1}\right)+\frac{k_{z} \sin \theta}{2 A_{3} \tilde{\omega}}, \\
& D_{6}=\frac{k_{z, e} \tilde{\omega} \mu \varepsilon_{e} \sqrt{\mu \varepsilon_{o}} R^{2}}{2 k_{z, o} k_{o} A_{2} A_{3}}\left(\frac{k_{o} \sin \theta}{\widetilde{\omega} \mu \varepsilon_{e} R} A_{3}-\frac{R \cos \theta}{\sqrt{\mu \varepsilon_{o}}} A_{1}\right)-\frac{k_{z} \sin \theta}{2 A_{3} \tilde{\omega}}, \\
& D_{7}=\frac{k_{z, e} \tilde{\omega} \mu \varepsilon_{e} \sqrt{\mu \varepsilon_{o}} R^{2}}{2 k_{z, o} k_{o} A_{2} A_{3}}\left(\frac{k_{o} k_{z} \cos \theta}{\widetilde{\omega}^{2} \mu \varepsilon_{e} R} A_{3}+\frac{R k_{z} \sin \theta}{\widetilde{\omega} \sqrt{\mu \varepsilon_{o}}} A_{1}\right)+\frac{\cos \theta}{2 A_{3}}, \\
& D_{8}=\frac{k_{z, e} \tilde{\omega} \mu \varepsilon_{e} \sqrt{\mu \varepsilon_{o}} R^{2}}{2 k_{z, o} k_{o} A_{2} A_{3}}\left(\frac{k_{o} k_{z} \cos \theta}{\widetilde{\omega}^{2} \mu \varepsilon_{e} R} A_{3}+\frac{R k_{z} \sin \theta}{\widetilde{\omega} \sqrt{\mu \varepsilon_{o}}} A_{1}\right)-\frac{\cos \theta}{2 A_{3}},
\end{aligned}
$$

where the parameters $A_{i}(i=1,2,3)$ are given as

$$
\begin{aligned}
& A_{1}=\frac{\kappa^{2} \sin \theta \cos \theta}{k_{o} \sqrt{\kappa^{2} \cos ^{2} \theta+k_{z, o}^{2}}}, \\
& A_{2}=\frac{k_{z, e}}{\sqrt{\kappa^{2} \cos ^{2} \theta+k_{z, e}^{2}}}, \\
& A_{3}=\frac{\sqrt{\kappa^{2} \cos ^{2} \theta+k_{z, o}^{2}}}{k_{o}} .
\end{aligned}
$$

\section{B. Definitions of Parameters $V_{i}$ in (39)}

The parameters come from the processes of solving equation groups (38), and they can relate to the coefficients of them as

$$
\begin{aligned}
& V_{1}=\frac{-\tilde{\omega} \mu\left(\varepsilon_{1}^{2} X_{1}^{2}+\varepsilon_{2}^{2}+\varepsilon_{3}^{2} Z_{1}^{2}\right)^{1 / 2} i 4 \pi \tilde{\omega} \mu X_{2}}{k_{1}\left(X_{1}-X_{2}\right)}, \\
& V_{2}=\frac{\tilde{\omega} \mu\left(\varepsilon_{1}^{2} X_{1}^{2}+\varepsilon_{2}^{2}+\varepsilon_{3}^{2} Z_{1}^{2}\right)^{1 / 2} i 4 \pi \tilde{\omega} \mu}{k_{1}\left(X_{1}-X_{2}\right)},
\end{aligned}
$$

$$
\begin{aligned}
& V_{3}=\frac{-i 4 \pi \kappa \tilde{\omega} \mu\left(\varepsilon_{1}^{2} X_{1}^{2}+\varepsilon_{2}^{2}+\varepsilon_{3}^{2} Z_{1}^{2}\right)^{1 / 2}\left(\cos \theta+X_{1} \sin \theta\right)}{k_{1} \varepsilon_{3}\left(X_{1}-X_{2}\right)}, \\
& V_{4}=\frac{-\tilde{\omega} \mu\left(\varepsilon_{1}^{2} X_{2}^{2}+\varepsilon_{2}^{2}+\varepsilon_{3}^{2} Z_{2}^{2}\right)^{1 / 2} i 4 \pi \mu \tilde{\omega} X_{1}}{k_{2}\left(X_{2}-X_{1}\right)}, \\
& V_{5}=\frac{\tilde{\omega} \mu\left(\varepsilon_{1}^{2} X_{2}^{2}+\varepsilon_{2}^{2}+\varepsilon_{3}^{2} Z_{2}^{2}\right)^{1 / 2} i 4 \pi \mu \tilde{\omega}}{k_{2}\left(X_{2}-X_{1}\right)}, \\
& V_{6}=\frac{-i 4 \pi \kappa \tilde{\omega} \mu\left(\varepsilon_{1}^{2} X_{2}^{2}+\varepsilon_{2}^{2}+\varepsilon_{3}^{2} Z_{2}^{2}\right)^{1 / 2}\left(\cos \theta+X_{1} \sin \theta\right)}{\varepsilon_{3} k_{2}\left(X_{2}-X_{1}\right)} \\
& V_{7}=\frac{-i 4 \pi \tilde{\omega} \mu N_{2}\left(\kappa \sin \theta Z_{1}-k_{z, 1}\right)}{\mathcal{A}}, \\
& V_{8}=\frac{-i 4 \pi \tilde{\omega} \mu N_{2}\left(k_{z, 1} X_{1}-Z_{1} \kappa \cos \theta\right)}{\mathcal{A}}, \\
& V_{9}=\frac{N_{2}\left[i 4 \pi \mu \kappa \sin \theta\left(\kappa \sin \theta Z_{1}-k_{z, 1}\right)-\mathscr{B}\right]}{\mathcal{A}}, \\
& V_{10}=\frac{-i 4 \pi \tilde{\omega} \mu N_{1}\left(\kappa \sin \theta Z_{2}-k_{z, 2}\right)}{\mathcal{C}}, \\
& V_{11}=\frac{-i 4 \pi \tilde{\omega} \mu N_{1}\left(k_{z, 2} X_{2}-Z_{2} \kappa \cos \theta\right)}{\mathcal{C}}, \\
& N_{1}\left[i 4 \pi \mu \kappa \sin \theta\left(\kappa \sin \theta Z_{2}-k_{z, 2}\right)-\mathscr{D}\right] \\
& \mathcal{C}
\end{aligned}
$$

where $\mathcal{A}$ denotes $\left(\kappa \sin \theta Z_{2}-k_{z, 2}\right)\left(k_{z, 1} X_{1}-Z_{1} \kappa \cos \theta\right)-$ $\left(k_{z, 2} X_{2}-Z_{2} \kappa \cos \theta\right)\left(\kappa \sin \theta Z_{1}-k_{z, 1}\right), \quad \mathscr{B}$ denotes $i 4 \pi \mu \kappa \cos \theta\left(k_{z, 1} X_{1}-Z_{1} \kappa \cos \theta\right), e$ denotes $\left(\kappa \sin \theta Z_{1}-\right.$ $\left.k_{z, 1}\right)\left(k_{z, 2} X_{2}-Z_{2} \kappa \cos \theta\right)-\left(k_{z, 1} X_{1}-Z_{1} \kappa \cos \theta\right)\left(\kappa \sin \theta Z_{2}-k_{z, 2}\right)$, and $\mathscr{D}$ denotes $i 4 \pi \mu \kappa \cos \theta\left(k_{z, 2} X_{2}-Z_{2} \kappa \cos \theta\right)$.

\section{Definitions of Parameters $U_{i}$ in (42)}

The parameters $U_{i}$ are gotten in the processes of solving equation groups (40) and (41), and they can be related to the coefficients in front of the field amplitudes. They firstly relate to parameters $I_{i}^{\prime}, i=1,2, \ldots, 6$, and $F_{i}^{\prime}, i=1,2, \ldots, 9$, which will defined later:

$$
\begin{aligned}
& U_{1}=\frac{I_{3}^{\prime} I_{5}^{\prime}-I_{2}^{\prime} I_{6}^{\prime}}{I_{1}^{\prime} I_{5}^{\prime}-I_{2}^{\prime} I_{4}^{\prime}} e^{i k_{z, 1} h}, \\
& U_{2}=\frac{F_{1}^{\prime} I_{5}^{\prime}-F_{4}^{\prime} I_{2}^{\prime}}{F_{7}\left(I_{1}^{\prime} I_{5}^{\prime}-I_{2}^{\prime} I_{4}^{\prime}\right)} e^{-i k_{z, 1} h}, \\
& U_{3}=\frac{F_{1}^{\prime} I_{4}^{\prime}-F_{4}^{\prime} I_{1}^{\prime}}{F_{7}^{\prime}\left(I_{2}^{\prime} I_{4}^{\prime}-I_{1}^{\prime} I_{5}^{\prime}\right)} e^{-i k_{z, 1} h}, \\
& U_{4}=\frac{I_{2}^{\prime}}{I_{1}^{\prime} I_{5}^{\prime}-I_{2}^{\prime} I_{4}^{\prime}}, \\
& U_{5}=\frac{I_{5}^{\prime}}{I_{1}^{\prime} I_{5}^{\prime}-I_{2}^{\prime} I_{4}^{\prime}} e^{-i k_{z, 1} h}, \\
& U_{6}=\frac{I_{1}^{\prime}}{I_{2}^{\prime} I_{4}^{\prime}-I_{1}^{\prime} I_{5}^{\prime}} e^{i k_{z, 1} h}, \\
& U_{7}=\frac{I_{4}^{\prime}}{I_{2}^{\prime} I_{4}^{\prime}-I_{1}^{\prime} I_{5}^{\prime}} e^{-i k_{z, 1} h} .
\end{aligned}
$$


The parameters $I_{i}^{\prime}, i=1,2, \ldots, 6$, are given as functions of $F_{i}^{\prime}, i=1,2, \ldots, 9$, and $Q_{i}, i=1,2, \ldots, 8$

$$
\begin{aligned}
& I_{1}^{\prime}=F_{2}^{\prime}-\frac{F_{1}^{\prime} F_{8}^{\prime}}{F_{7}^{\prime}}, \\
& I_{2}^{\prime}=F_{3}^{\prime}-\frac{F_{1}^{\prime} F_{9}^{\prime}}{F_{7}^{\prime}}, \\
& I_{3}^{\prime}=\frac{F_{1}^{\prime} Q_{1}}{F_{7}^{\prime} Q_{3}}+\frac{Q_{5}}{Q_{3}}, \\
& I_{4}^{\prime}=F_{5}^{\prime}-\frac{F_{4}^{\prime} F_{8}^{\prime}}{F_{7}^{\prime}}, \\
& I_{5}^{\prime}=F_{6}^{\prime}-\frac{F_{4}^{\prime} F_{9}^{\prime}}{F_{7}^{\prime}}, \\
& I_{6}^{\prime}=\frac{F_{4}^{\prime} Q_{1}}{F_{7}^{\prime} Q_{3}}-\frac{Q_{7}}{Q_{3}} .
\end{aligned}
$$

The parameters $F_{i}^{\prime}, i=1,2, \ldots, 9$, are defined as the functions of $Q_{i}, i=1,2, \ldots, 8$,

$$
\begin{aligned}
& F_{1}^{\prime}=\frac{Q_{4} Q_{5}}{Q_{3}}+Q_{6}, \\
& F_{2}^{\prime}=Q_{7} e^{-i k_{z, 2} h}+\frac{Q_{5} Q_{1}}{Q_{3}} e^{i k_{z, 1} h}, \\
& F_{3}^{\prime}=Q_{8} e^{-i k_{z, 2} h}-\frac{Q_{5} Q_{2}}{Q_{3}} e^{i k_{z, 1} h}, \\
& F_{4}^{\prime}=-\frac{Q_{7} Q_{4}}{Q_{3}}-Q_{8}, \\
& F_{5}^{\prime}=-Q_{5} e^{i k_{z, 2} h}-\frac{Q_{7} Q_{1}}{Q_{3}} e^{i k_{z, 1} h}, \\
& F_{6}^{\prime}=-Q_{6} e^{i k_{z, 2} h}+\frac{Q_{7} Q_{2}}{Q_{3}} e^{i k_{z, 1} h}, \\
& F_{7}^{\prime}=Q_{2}-\frac{Q_{1} Q_{4}}{Q_{3}}, \\
& F_{8}^{\prime}=Q_{3} e^{-i k_{z, 1} h}-\frac{Q_{1}^{2}}{Q_{3}} e^{i k_{z, 1} h}, \\
& F_{9}^{\prime}=-Q_{4} e^{-i k_{z, 1} h}+\frac{Q_{1} Q_{2}}{Q_{3}} e^{i k_{z, 1} h} .
\end{aligned}
$$

The parameters $Q_{i}, i=1,2, \ldots, 8$, relate with the coefficients in (40) and (41) directly:

$$
\begin{aligned}
Q_{1}= & {\left[\frac{\left[\left(X_{1} \cos \theta+\sin \theta\right) X_{2}+\left(X_{2}-X_{1}\right) \sin \theta\right]}{2 S_{5}\left(X_{2}-X_{1}\right) X_{1}}\right.} \\
& \left.+\frac{\left(S_{4} \cos \theta-S_{2} \sin \theta\right) k_{z}}{2\left(S_{2} S_{3}-S_{1} S_{4}\right) \tilde{\omega}}\right], \\
Q_{2}= & \frac{-\left(S_{4} \sin \theta+S_{2} \cos \theta\right)}{2\left(S_{2} S_{3}-S_{1} S_{4}\right)} \\
& \left.+\frac{\left[\left(X_{1} \sin \theta-\cos \theta\right) X_{2}+\left(X_{2}-X_{1}\right) \cos \theta\right]}{2 S_{5}\left(X_{2}-X_{1}\right) X_{1}} \frac{k_{z}}{\widetilde{\omega}}\right], \\
Q_{3}= & \frac{\left[\left(X_{2}-X_{1}\right) \sin \theta-X_{2}\left(\sin \theta+\cos \theta X_{1}\right)\right]}{2 S_{5} X_{1}\left(X_{2}-X_{1}\right)} \\
& \left.-\frac{\left(S_{4} \cos \theta-S_{2} \sin \theta\right)}{2\left(S_{2} S_{3}-S_{1} S_{4}\right)} \frac{k_{z}}{\widetilde{\omega}}\right], \\
Q_{4}= & \frac{\left[\cos \theta\left(X_{2}-X_{1}\right)-X_{2}\left(\cos \theta-X_{1} \sin \theta\right)\right]}{2 S_{5} X_{1}\left(X_{2}-X_{1}\right)} \frac{k_{z}}{\widetilde{\omega}} \\
& \left.+\frac{\left(S_{4} \sin \theta+S_{2} \cos \theta\right)}{2\left(S_{2} S_{3}-S_{1} S_{4}\right)}\right]
\end{aligned}
$$

$$
\begin{aligned}
& Q_{5}=- {\left[\frac{\left[-\left(S_{2} S_{3}-S_{1} S_{4}\right) \cos \theta-S_{1}\left(S_{4} \cos \theta-S_{2} \sin \theta\right)\right]}{2\left(S_{2} S_{3}-S_{1} S_{4}\right) S_{2}} \frac{k_{z}}{\widetilde{\omega}}\right.} \\
&\left.+\frac{\left(X_{1} \cos \theta+\sin \theta\right)}{2 S_{6}\left(X_{2}-X_{1}\right)}\right], \\
& Q_{6}= {\left[\frac{\left(\cos \theta-X_{1} \sin \theta\right)}{2 S_{6}\left(X_{2}-X_{1}\right)} \frac{k_{z}}{\widetilde{\omega}}\right.} \\
&\left.+\frac{\left[\left(S_{2} S_{3}-S_{1} S_{4}\right) \sin \theta+S_{1}\left(S_{4} \sin \theta+S_{2} \cos \theta\right)\right]}{2\left(S_{2} S_{3}-S_{1} S_{4}\right) S_{2}}\right], \\
& Q_{7}=\left[\frac{\left[\cos \theta\left(S_{2} S_{3}-S_{1} S_{4}\right)+S_{1}\left(S_{4} \cos \theta-S_{2} \sin \theta\right)\right]}{2 S_{2}\left(S_{2} S_{3}-S_{1} S_{4}\right)} \frac{k_{z}}{\widetilde{\omega}}\right. \\
&\left.+\frac{\left(\sin \theta+\cos \theta X_{1}\right)}{2 S_{6}\left(X_{2}-X_{1}\right)}\right], \\
& Q_{8}= {\left[\frac{\left[\sin \theta\left(S_{2} S_{3}-S_{1} S_{4}\right)+S_{1}\left(S_{4} \sin \theta+S_{2} \cos \theta\right)\right]}{2 S_{2}\left(S_{2} S_{3}-S_{1} S_{4}\right)}\right.} \\
&\left.-\frac{\left(\cos \theta-X_{1} \sin \theta\right)}{2 S_{6}\left(X_{2}-X_{1}\right)} \frac{k_{z}}{\widetilde{\omega}}\right] .
\end{aligned}
$$

\section{Acknowledgments}

W. Wen would like to thank the support for this work by the Hong Kong RGC Grant HKUST2/CRF/11G. B. Hou would like to acknowledge the support from the National Natural Science Foundation of China (Grant no. 11104198) and from a Project Funded by the Priority Academic Program Development of Jiangsu Higher Education Institutions (PAPD).

\section{References}

[1] B. Hou, H. Wen, Y. Leng, and W. Wen, "Enhanced transmission of electromagnetic waves through metamaterials," Applied Physics A, vol. 87, no. 2, pp. 217-221, 2007.

[2] X. Xiao, X. Yi, B. Hou et al., "Subwavelength waveguiding and imaging with a one-dimensional array of metallic H-fractals," New Journal of Physics, vol. 12, Article ID 073021, 2010.

[3] V. M. Shalaev, "Optical negative-index metamaterials," Nature Photonics, vol. 1, no. 1, pp. 41-48, 2007.

[4] D. R. Smith, J. B. Pendry, and M. C. K. Wiltshire, "Metamaterials and negative refractive index," Science, vol. 305, no. 5685, pp. 788-792, 2004.

[5] B. Hou and W. Wen, "Transmission resonances of electromagnetic wave through metallic gratings: phase and field characterizations," Optics Express, vol. 16, no. 21, pp. 1709817106, 2008.

[6] X. Xiao, J. Wu, F. Miyamaru et al., "Fano effect of metamaterial resonance in terahertz extraordinary transmission," Applied Physics Letters, vol. 98, no. 1, Article ID 011911, 2011.

[7] N. I. Zheludev, "The road ahead for metamaterials," Science, vol. 328, no. 5978, pp. 582-583, 2010.

[8] D. R. Smith, S. Schultz, P. Markoš, and C. M. Soukoulis, "Determination of effective permittivity and permeability of metamaterials from reflection and transmission coefficients," Physical Review B, vol. 65, no. 19, Article ID 195104, 2002.

[9] A. M. Bratkovsky, E. Ponizovskaya, E. Kim et al., "Properties of nanostructured metamaterials at optical frequencies," in 
Optoelectronic Materials and Devices III, vol. 7135, 71352K of Proceedings of SPIE, Hangzhou, China, October 2008.

[10] C. Fu and Z. M. Zhang, "Thermal radiative properties of metamaterials and other nanostructured materials: a review," Frontiers of Energy and Power Engineering in China, vol. 3, no. 1, pp. 11-26, 2009.

[11] A. V. Kabashin, P. Evans, S. Pastkovsky et al., "Plasmonic nanorod metamaterials for biosensing," Nature Materials, vol. 8, no. 11 , pp. 867-871, 2009.

[12] C. R. Simovski and S. A. Tretyakov, "On effective electromagnetic parameters of artificial nanostructured magnetic materials," Photonics and Nanostructures, vol. 8, no. 4, pp. 254263, 2010.

[13] W. T. Lu and S. Sridhar, "Superlens imaging theory for anisotropic nanostructured metamaterials with broadband all-angle negative refraction," Physical Review B, vol. 77, no. 23, Article ID 233101, 2008.

[14] K. Joulain, J. P. Mulet, F. Marquier, R. Carminati, and J. J. Greffet, "Surface electromagnetic waves thermally excited: radiative heat transfer, coherence properties and Casimir forces revisited in the near field," Surface Science Reports, vol. 57, no. 3-4, pp. 59-112, 2005.

[15] R. Carminati and J.-J. Greffet, "Near-field effects in spatial coherence of thermal sources," Physical Review Letters, vol. 82, no. 8, pp. 1660-1663, 1999.

[16] W. T. Lau, J.-T. Shen, G. Veronis, and S. Fan, "Spatial coherence of the thermal electromagnetic field in the vicinity of a dielectric slab," Physical Review E, vol. 76, no. 1, Article ID 016601, 2007.

[17] J. E. Sipe, "New Green-function formalism for surface optics," Journal of the Optical Society of America B, vol. 4, no. 4, pp. 481-489, 1987.

[18] F. S. S. Rosa, D. A. R. Dalvit, and P. W. Milonni, "Casimirlifshitz theory and metamaterials," Physical Review Letters, vol. 100, no. 18, Article ID 183602, 2008.

[19] J. Hao, Y. Yuan, L. Ran et al., "Manipulating electromagnetic wave polarizations by anisotropic metamaterials," Physical Review Letters, vol. 99, no. 6, Article ID 063908, 2007.

[20] J. Hao, Q. Ren, Z. An et al., "Optical metamaterial for polarization control," Physical Review A, vol. 80, no. 2, Article ID 023807, 2009.

[21] F. J. García-Vidal, L. Martín-Moreno, H. J. Lezec, and T. W. Ebbesen, "Focusing light with a single subwavelength aperture flanked by surface corrugations," Applied Physics Letters, vol. 83, no. 22, pp. 4500-4502, 2003.

[22] L. Martín-Moreno, F. J. Garcia-Vidal, H. J. Lezec, A. Degiron, and T. W. Ebbesen, "Theory of highly directional emission from a single subwavelength aperture surrounded by surface corrugations," Physical Review Letters, vol. 90, no. 16, Article ID 167401, 2003.

[23] J. Hao and L. Zhou, "Electromagnetic wave scatterings by anisotropic metamaterials: generalized $4 \times 4$ transfer-matrix method," Physical Review B, vol. 77, no. 9, Article ID 094201, 2008. 

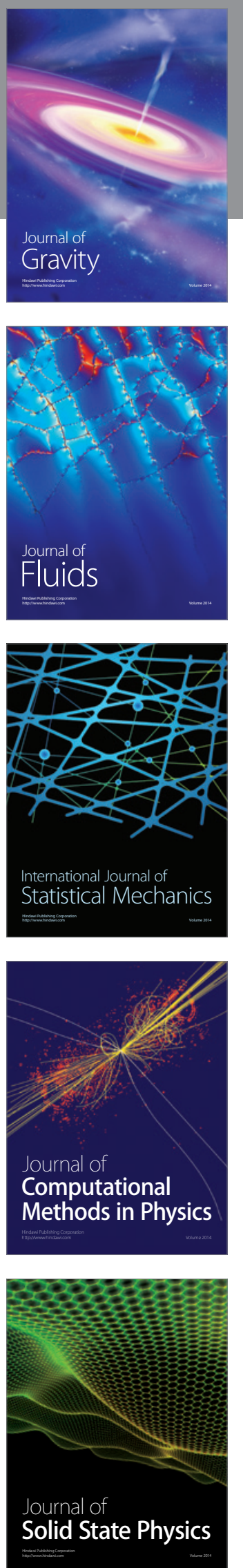

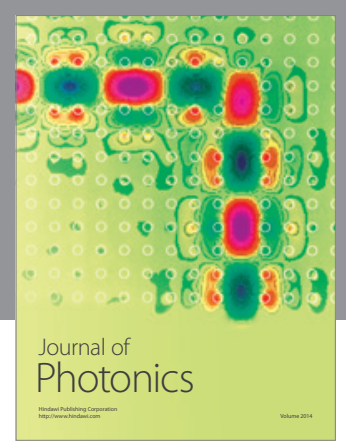

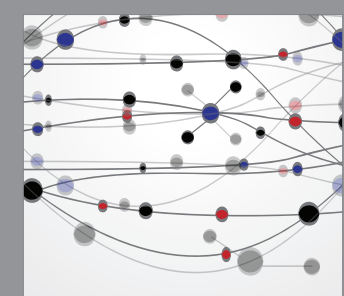

The Scientific World Journal
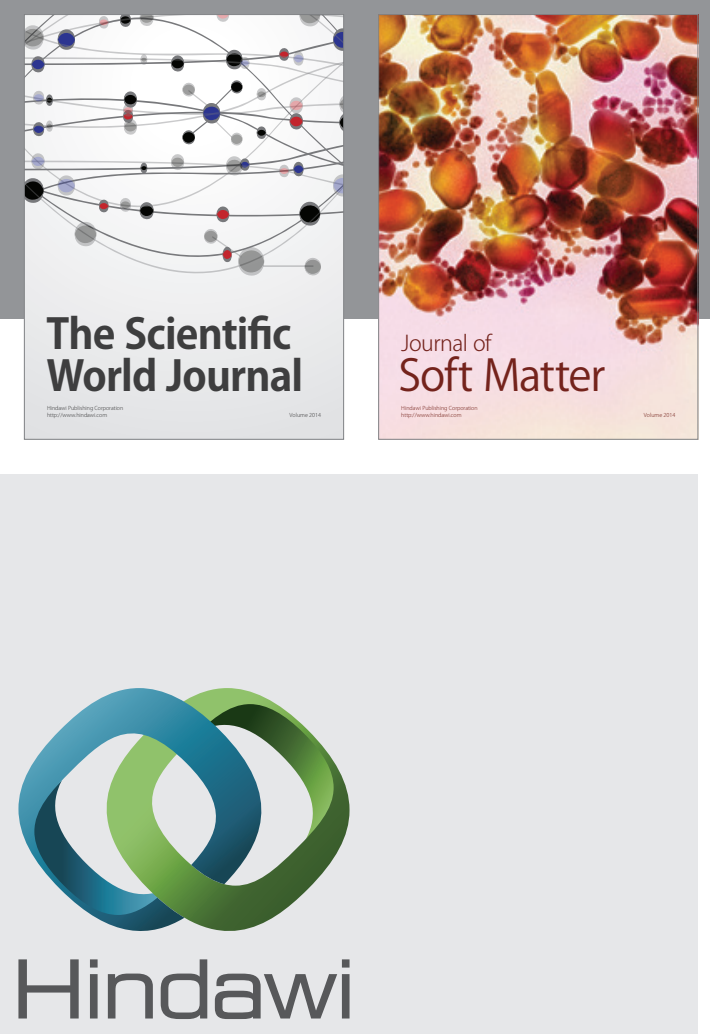

Submit your manuscripts at

http://www.hindawi.com
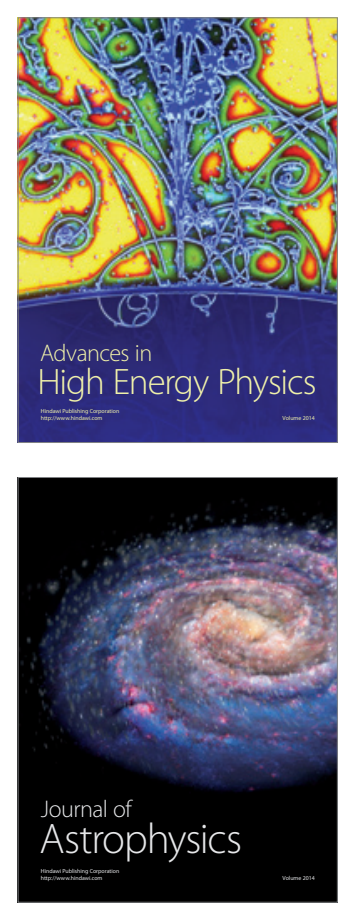
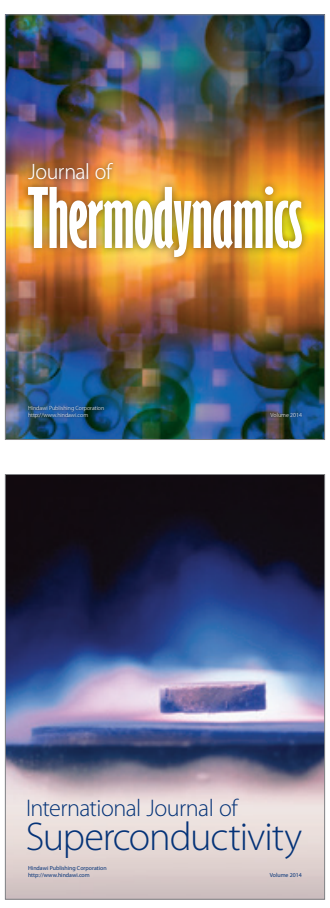
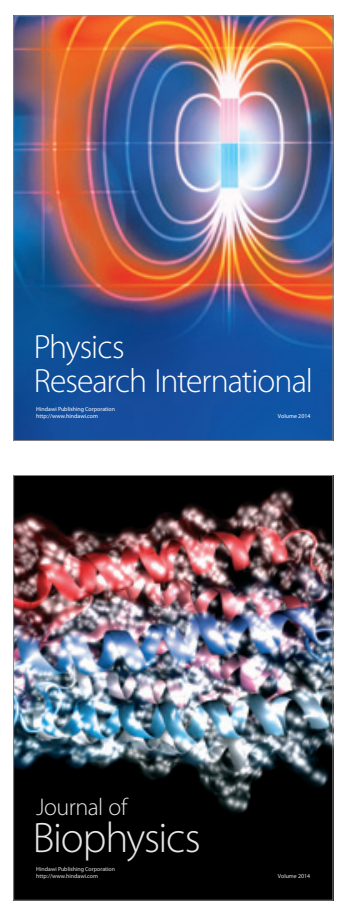
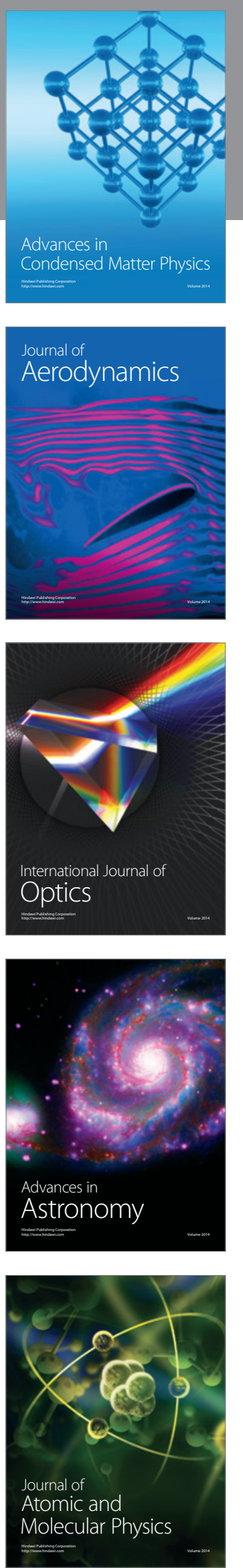